\title{
Outflows from the high-mass protostars NGC 7538 IRS1/2 observed with bispectrum speckle interferometry ${ }^{\star}$
}

\section{Signatures of flow precession}

\author{
S. Kraus ${ }^{1}$, Y. Balega ${ }^{2}$, M. Elitzur ${ }^{3}$, K.-H. Hofmann ${ }^{1}$, Th. Preibisch ${ }^{1}$, \\ A. Rosen ${ }^{1}$, D. Schertl ${ }^{1}$, G. Weigelt ${ }^{1}$, and E. T. Young ${ }^{4}$ \\ 1 Max Planck Institut für Radioastronomie, Auf dem Hügel 69, 53121 Bonn, Germany \\ e-mail: skraus@mpifr-bonn.mpg.de \\ 2 Special Astrophysical Observatory, Russian Academy of Sciences, Nizhnij Arkhyz, Zelenchuk region, Karachai-Cherkesia, 357147 , \\ Russia \\ 3 Department of Physics \& Astronomy, University of Kentucky, Lexington, KY 40506, USA \\ 4 Steward Observatory, University of Arizona, 933 North Cherry Avenue, Tucson, AZ 85721, USA
}

Received 22 February 2006 / Accepted 10 April 2006

\begin{abstract}
Context. NGC 7538 IRS1 is a high-mass $\left(30 M_{\odot}\right)$ protostar with a CO outflow, an associated ultracompact $\mathrm{H}$ II region, and a linear methanol maser structure, which might trace a Keplerian-rotating circumstellar disk. The directions of the various associated axes are misaligned with each other.

Aims. We investigate the near-infrared morphology of the source to clarify the relations among the various axes.

Methods. $K^{\prime}$-band bispectrum speckle interferometry was performed at two 6-meter-class telescopes - the BTA $6 \mathrm{~m}$ telescope and the $6.5 \mathrm{~m}$ MMT. Complementary IRAC images from the Spitzer Space Telescope Archive were used to relate the structures detected with the outflow at larger scales.

Results. High-dynamic range images show fan-shaped outflow structure in which we detect 18 stars and several blobs of diffuse emission. We interpret the misalignment of various outflow axes in the context of a disk precession model, including numerical hydrodynamic simulations of the molecular emission. The precession period is $\sim 280$ years and its half-opening angle is $\sim 40^{\circ}$. A possible triggering mechanism is non-coplanar tidal interaction of an (undiscovered) close companion with the circumbinary protostellar disk. Our observations resolve the nearby massive protostar NGC 7538 IRS2 as a close binary with separation of 195 mas. We find indications for shock interaction between the outflow activities in IRS1 and IRS2. Finally, we find prominent sites of star formation at the interface between two bubble-like structures in NGC 7538, suggestive of a triggered star formation scenario.

Conclusions. Indications of outflow precession have been discovered to date in a number of massive protostars, all with large precession angles $\left(\sim 20-45^{\circ}\right)$. This might explain the difference between the outflow widths in low- and high-mass stars and add support to a common collimation mechanism.
\end{abstract}

Key words. stars: formation - stars: individual: NGC 7538 IRS1 - stars: individual: NGC 7538 IRS2 - techniques: interferometric stars: winds, outflows - hydrodynamics

\section{Introduction}

Protostellar disks and outflows are essential constituents of the star formation process. For high-mass protostellar objects (HMPOs), direct evidence for the presence of compact circumstellar disks is still rare, whereas outflows seem to be omnipresent in the high-mass star forming regions. Outflows remove not only angular momentum from the infalling matter, but also help to overcome the radiation pressure limit to protostellar accretion, by carving out optically thin cavities along which the radiation pressure can escape (Krumholz et al. 2005).

^ Observations reported here were obtained at the MMT Observatory, a joint facility of the Smithsonian Institution and the University of Arizona, and at the BTA $6 \mathrm{~m}$ telescope of the Special Astrophysical Observatory, Russia. In addition, this work is based in part on archival data obtained with the Spitzer Space Telescope, which is operated by the Jet Propulsion Laboratory, California Institute of Technology under a contract with NASA.
How outflows are collimated is a matter of ongoing debate and may depend on the stellar mass of the outflow-driving source. One of the arguments in support of this conclusion is that outflows from high-mass stars appear less collimated than the outflows and jets from their low-mass counterparts (Wu et al. 2004). Therefore, it has been suggested that outflows from HMPOs might be driven by strong stellar winds, lacking a recollimation mechanism. Since HMPOs typically form in dense clusters, another possibility is confusion by the presence of multiple collimated outflows.

However, since there is evidence that the binary frequency is significantly higher for high-mass than for low-mass stars (e.g., Preibisch et al. 1999), another possibility is that outflows from HMPOs simply appear wider, assuming they undertake precession. A few cases where outflow precession have been proposed for HMPO outflows (e.g. Shepherd et al. 2000; Weigelt et al. $2002,2005)$ show precession angles of $\sim 20$ to $45^{\circ}$; considerably wider than the jet precession angles of typically just a few 
degrees observed towards low-mass stars (Terquem et al. 1999). This is in agreement with the general picture that high-mass stars form at high stellar density sites and therefore experience strong tidal interaction from close companions and stellar encounters.

The detection of precessing jet-driven outflows from HMPOs adds support to the hypothesis of a common formation mechanism for outflows from low to high-mass stars. Furthermore, jet precession carries information about the accretion properties of the driving source and, simultaneously, about the kinematics and stellar population within its closest vicinity, yielding a unique insight into the crowded places where highmass star formation occurs.

In this paper, we report another potential case of outflow precession concerning the outflow from the high-mass $\left(30 \mathrm{M}_{\odot}\right.$, Pestalozzi et al. 2004) protostellar object NGC 7538 IRS1.

We obtained bispectrum speckle interferometry of IRS1 and IRS2, which provides us with the spatial resolution to study the inner parts of the outflow, detecting filigreed fine structure within the flow. Information about even smaller scales is provided by the intriguing methanol maser feature, which was detected at the position of this infrared source and which was modeled successfully as a protostellar disk in Keplerian rotation (Pestalozzi et al. 2004). To search for outflow tracers on larger scales, we also present archival Spitzer/IRAC images. In addition, this allows us to relate the sources studied with bispectrum speckle interferometry with the overall star forming region and we find new hints for triggered star formation in this region.

\subsection{Previous studies of NGC 7538}

The NGC 7538 molecular cloud is located in the Cas OB2 association in the Perseus spiral arm at a distance of $\sim 2.8 \mathrm{kpc}$ (Blitz et al. 1982). Several authors noted that NGC 7538 might present a case of triggered or induced star formation since it shows ongoing star formation at various evolutionary stages, apparently arranged in a northwest (most developed) to southeast (youngest evolutionary stage) gradient (McCaughrean et al. 1991).

At optical wavelengths, the appearance of the region is dominated by diffuse $\mathrm{H}$ II emission, which extends several arcminutes from the southeast to the northwest (Lynds \& O'Neil 1986). In 1974, Wynn-Williams, Becklin, \& Neugebauer detected eleven infrared sources (IRS1-11) in the NGC 7538 region, wherein IRS1-3 are located on the southeast-corner of the fan-shaped $\mathrm{H}$ II emission in a small cluster of OB-stars. IRS 1 is the brightest NIR source within this cluster and is embedded within an ultracompact (UC) H II region whose size was estimated to be $\sim 0.4\left(n_{\mathrm{e}} \approx 10^{5} \mathrm{~cm}^{-3}\right.$, measured in 5 and $15 \mathrm{GHz} \mathrm{CO}$ continuum, Campbell 1984). The spectral type was estimated to be 07 (Akabane \& Kuno 2005), which implies a luminosity $\sim 9.6 \times 10^{4} L_{\odot}$. VLA observations with a resolution down to 0 !' $1^{\prime}$ (=180 AU) also revealed a double-peaked structure of ionized gas within the UC core (peaks separated by $\sim 00^{\prime \prime} 2$ ), which was interpreted as a disk collimating a north-south-oriented outflow (Campbell 1984; Gaume et al. 1995). This interpretation is also supported by the detection of elongation of the dust-emitting region at mid-infrared (MIR) wavelengths (5 $\mu \mathrm{m}$ : Hackwell et al. $1982 ; 11.7 \mu \mathrm{m}$ and $18.3 \mu \mathrm{m}$ : De Buizer \& Minier 2005) and imaging studies performed in the sub-millimeter continuum ( $350 \mu \mathrm{m}, 450 \mu \mathrm{m}, 800 \mu \mathrm{m}, 850 \mu \mathrm{m}, 1.3 \mathrm{~mm}$ : Sandell \& Sievers 2004 , showing an elliptical source with a size of $\sim 11$.' $6 \times 7$ ".' 6 along $\mathrm{PA}^{1} \sim-80^{\circ}$ ) and CO line emission (Scoville et al. 1986,

1 Following the convention, we measure the position angle (PA) from north to east. showing a disk-like structure extending $\sim 22^{\prime \prime}$ in the east-west direction). Also, polarization measurements of the infrared emission around IRS1 can be construed in favor of the disk interpretation (Dyck \& Capps 1978; Tamura et al. 1991). Kawabe et al. (1992) carried out interferometric CS $(J=2 \rightarrow 1)$ observations and found a ring-like structure, which they interpret as a nearly face-on protostellar disk of dense molecular gas.

Further evidence for outflow activity was found by Gaume et al. (1995), who measured the profile of the H66 $\alpha$ recombination line and derived high velocities of $250 \mathrm{~km} \mathrm{~s}^{-1}$, indicating a strong stellar outflow from IRS1. CO $(J=1 \rightarrow 0)$ spectral line mapping showed a bipolar flow (Fischer et al. 1985). The mass outflow rate $\dot{M}_{\text {outflow }}$ from IRS1 was estimated to be $\sim 5.4 \times 10^{-3} M_{\odot} \mathrm{yr}^{-1}$ (Davis et al. 1998). Interferometric observations by Scoville et al. (1986, beam size $7^{\prime \prime}$ ) show that the blue and red-shifted lobes are separated by $28^{\prime \prime}$ with a position angle of $-45^{\circ}$, and IRS1 is located on this axis just between the lobes of this high-velocity $\left(-76\right.$ to $\left.-37 \mathrm{~km} \mathrm{~s}^{-1}\right) \mathrm{CO}$ outflow. In comparing the data obtained with various beam sizes (Campbell 1984; Kameya et al. 1989), these seem to indicate a change in the position angle of the flow direction at different spatial scales, ranging from $\mathrm{PA} \sim 0^{\circ}$ at $0 !^{\prime} 3, \mathrm{PA} \sim-25^{\circ}$ at $2^{\prime \prime}, \mathrm{PA} \sim-35^{\circ}$ at $7^{\prime \prime}$, to PA $-40^{\circ}$ at $16^{\prime \prime}$.

Within the immediate $(\sim 0,5)$ vicinity of IRS1, a large variety of masers has been discovered, including $\mathrm{OH}$ (Dickel et al. 1982), $\mathrm{H}_{2} \mathrm{CO}$ (formaldehyde, Rots et al. 1981; Hoffman et al. 2003), $\mathrm{NH}_{3}$ (ammonia, Madden et al. 1986), $\mathrm{CH}_{3} \mathrm{OH}$ (methanol, five features A, B, C, D, E were detected at 6.7 and 12.2 GHz: Menten et al. 1986; Minier et al. 1998, 2000), ${ }^{15} \mathrm{NH}_{3}$ (Johnston et al. 1989), and $\mathrm{H}_{2} \mathrm{O}$ (Kameya et al. 1990). Some of the masers show only vague signs for a systematic alignment within linear $\left({ }^{15} \mathrm{NH}_{3}, \mathrm{PA} \sim-60^{\circ}\right)$ or ring-like structures $\left(\mathrm{H}_{2} \mathrm{O}\right.$, methanol-maser feature E). However, the methanol-maser feature A represents one of the most convincing cases of systematic alignment, in both linear spatial arrangement $\left(\mathrm{PA} \sim-62^{\circ}\right)$ and well-defined velocity gradient, observed to date in any maser source. The qualitative interpretation of this structure as an edgeon circumstellar disk (Minier et al. 1998) was later confirmed by the detailed modeling of Pestalozzi et al. (2004), which showed that the alignment in the position-line-of-sight (LOS) velocity diagram of maser feature A can be modeled accurately assuming a protostellar disk with Keplerian rotation.

Aiming for a more complete picture, several authors (e.g. Minier et al. 1998; De Buizer \& Minier 2005) also tried to incorporate the presence of methanol maser features B, C, D, and E in the circumstellar disk model for feature $\mathrm{A}$ and interpreted them as part of an outflow which is oriented perpedicular to feature A. Since these maser features are southwards of the putative circumstellar disk, it remains unclear why they appear blue-shifted with respect to feature A (Minier et al. 1998), whereas the southern lobe of the CO-outflow is red-shifted.

Besides the circumstellar disk interpretation for the origin of the maser feature A mentioned above, an alternative scenario was proposed by De Buizer \& Minier (2005), who suggested that feature A might trace the walls of an outflow cavity.

The region was also intensively observed in the infrared. Survey images of the infrared continuum emission were presented by Campbell \& Persson $(1988, H, K)$ and Ojha et al. (2004, J, H, $K_{\mathrm{s}}$ ) and showed diffuse emission, which extends from the IRS1-3 cluster in a fan-shaped structure towards the northeast and north, approximately tracing the optical H II region. The northeast border of this NIR emitting region also appears very pronounced in the continuum-subtracted $\mathrm{H}_{2} 2.122 \mu \mathrm{m}$ maps by Davis et al. (1998), possibly tracing the illuminated 
surfaces of nearby molecular clouds or the inner walls of a vast outflow cavity. Furthermore, Davis et al. (1998) discovered two bowshock-shaped structures, centered roughly on the IRS1-3 cluster and orientated again along the northwestsoutheast direction $\left(\mathrm{PA} \sim-30^{\circ}\right.$ ) in $\mathrm{H}_{2} 2.122 \mu \mathrm{m}$. With imaging at arcsecond resolution and the use of several spectral filters $(J, H$, $K$, [Fe II] $1.65 \mu \mathrm{m}, \mathrm{Br} \gamma 2.165 \mu \mathrm{m}, \mathrm{H}_{2} 2.122 \mu \mathrm{m}$, and $\left.3.29 \mu \mathrm{m}\right)$, Bloomer et al. (1998) attempted to identify the source and mechanism of the outflow. Based on a cometary-shaped morphology in the [Fe II] line images and shell-like rings observed in the $J$, $H$, and $K$-bands, these authors propose a stellar wind bowshock model in which the motion of IRS2 relative to the molecular cloud produces the diffuse NIR emission within the vicinity of the IRS1-3 cluster.

The first $K$-band speckle images, taken with the $3.5 \mathrm{~m}$ telescope on Calar Alto were presented by Alvarez et al. (2004) and showed substructure in the vicinity of IRS1; namely, two strong blobs $\left(A, \mathrm{PA} \sim-45^{\circ} ; B, \mathrm{PA} \sim-70^{\circ}\right)$, a diffuse emission feature $\left(C, \mathrm{PA} \sim 0^{\circ}\right)$ as well as several faint point-like sources $(a-f)$.

\section{Observations}

\subsection{Bispectrum speckle interferometry}

The first set of observations was performed on 2002-09-24 using the $6.0 \mathrm{~m}$ BTA (Big Telescope Alt-azimuthal) telescope of the Special Astrophysical Observatory located on Mt. Pastukhov in Russia. Additional data were gathered 2004-12-20 with the MMT (Multiple Mirror Telescope) on Mt. Hopkins in Arizona, which harbors a $6.5 \mathrm{~m}$ primary mirror. As detector, we used at both telescopes one $512 \times 512$ pixel quadrant of the Rockwell HAWAII array in our speckle camera. All observations were carried out using a $K^{\prime}$-band filter centered on the wavelength $2.12 \mu \mathrm{m}$ with a bandwidth of $0.21 \mu \mathrm{m}$. During the BTA observation run, we recorded 420 speckle interferograms on NGC 7538 IRS1 and 400 interferograms on the unresolved star BSD 19-901 in order to compensate for the atmospheric speckle transfer function. The speckle interferograms of both objects were taken with an exposure time of $360 \mathrm{~ms}$ per frame. For the MMT observations, the star 2MASS 23134580+6124049 was used for the calibration and 120 (200) frames were recorded on the target (calibrator) with an $800 \mathrm{~ms}$ exposure time. The modulus of the Fourier transform of the object (visibility) was obtained with the speckle interferometry method (Labeyrie 1970). For image reconstruction we used the bispectrum speckle interferometry method (Weigelt 1977; Weigelt \& Wirnitzer 1983; Lohmann et al. 1983; Hofmann \& Weigelt 1986). With pixel sizes of 27.0 mas (BTA) and 28.7 mas (MMT) on the sky, the reconstructed images possess fields of views of 13." 8 (BTA) and 13.'1 (MMT), respectively.

We found that the BTA data allows the highest spatial resolution (and is therefore perfectly suited for the identification of point-sources within the field), whereas the image reconstructed from the MMT data allows a high dynamic range in the diffuse emission. Therefore, we show the diffuse emission within an image of moderate resolution (reconstructed from MMT data, see Fig. 1a) and perform point-source identifications within the higher resolution image reconstructed from BTA data (Fig. 1b). In order to distinguish point-sources and diffuse structures reliably, we reconstructed images of various resolutions (146 mas, 97 mas, 72 mas) and carefully examined changes in the peak brightness of the detected features. Whereas for point-sources the peak brightness increases systematically, it stays constant or decreases for diffuse structures.

To perform an absolute calibration of the astrometry in our images, we measured the position of IRS1 and IRS2 in the Two Micron All Sky Survey (2MASS) $K_{\mathrm{s}}$ Atlas images and use the determined absolute positions as reference for our astrometry. We estimate that the accuracy reached in the relative astrometry is $\sim 0$.' 1 . The absolute calibration introduces further errors $(\sim 0,2)$.

\subsection{Spitzer/IRAC archive data}

In order to relate our high-resolution images with the morphology of the NGC 7538 molecular cloud at large scales, we examined archival 3.6, 4.5, 5.8, and $8.0 \mu \mathrm{m}$ images (PI: G. G. Fazio), taken with the Infrared Array Camera (IRAC, Fazio et al. 2004) on the Spitzer Space Telescope. The four bands are recorded simultaneously using two InSb $(3.6 \mu \mathrm{m}, 4.5 \mu \mathrm{m})$ and two Si:As $(5.8 \mu \mathrm{m}, 8.0 \mu \mathrm{m})$ detectors. The central wavelengths and bandwidths of the IRAC bands (Hora et al. 2004) are $3.56 \mu \mathrm{m}$ $(\Delta \lambda=0.75 \mu \mathrm{m}), 4.52 \mu \mathrm{m}(\Delta \lambda=1.01 \mu \mathrm{m}), 5.73 \mu \mathrm{m}(\Delta \lambda=$ $1.42 \mu \mathrm{m})$, and $7.91 \mu \mathrm{m}(\Delta \lambda=2.93 \mu \mathrm{m})$. Each image consists of $256 \times 256$ pixels, corresponding to a $\sim 5^{\prime} \times 5^{\prime}$ field-of-view on the sky. The data used include 48 Spitzer pointings taken on 2003 December 23 in the High Dynamic Range (HDR) mode. In HDR mode, for each pointing, images are taken with two exposure times $(0.6 \mathrm{~s}$ and $12 \mathrm{~s})$ in order to record both bright and faint structures. However, the two brightest sources, IRS1 and IRS9, are saturated even within the $0.6 \mathrm{~s}$ exposure.

We used the mopex software (2005-09-05 version), released by the Spitzer Science Center (SSC), to process both the long and short exposure images. Beside the basic calibration steps applied by the Basic Calibrated Data (BCD) pipeline (S11.0.2), we performed Radhit detection, artifact masking, and pointing refinement. Finally we generated a mosaic in which the saturated pixels of the long exposure image were replaced by the corresponding pixels of the $0.6 \mathrm{~s}$ exposure. The optical design of IRAC induces a shift of $\sim 6.8$ between the $3.6 / 5.8 \mu \mathrm{m}$ and $4.5 / 8.0 \mu \mathrm{m}$ pointings, leaving an overlap of 5.11 between all four bands.

In Fig. 2, color composites of the 3.6/4.5/5.8 $\mu \mathrm{m}$ and 4.5/5.8/8.0 $\mu \mathrm{m}$ band images are shown.

The diffuse emission in three of the four IRAC bands is dominated by Polycyclic Aromatic Hydrocarbons (PAHs, Churchwell et al. 2004), which trace the border of regions excited by the UV photons from HMPOs particularly well. Contributions are also expected from several vibrational levels of $\mathrm{H}_{2}$ (Smith \& Rosen 2005b), atomic lines, $\mathrm{CO}$ vibrational bands, and thermal dust grain emission.

\section{Results}

\subsection{Bispectrum speckle interferometry: small-scale structures around IRS1/2}

\subsubsection{IRS1 Airy disk elongation and diffuse emission}

In our speckle images, the Airy disk of IRS1 itself appears asymmetric, being more extended towards the northwest direction (PA $\sim-70^{\circ}$, see Fig. $1 \mathrm{~b}$ and inset in the lower left of Fig. 3). In the same direction (PA $\sim-60^{\circ}$ ), we find two strong blobs $\left(A, B+B^{\prime}\right)$ of diffuse emission at separations of $\sim 1^{\prime \prime}$ and $2^{\prime \prime}$. These blobs and additional diffuse emission seem to form a 


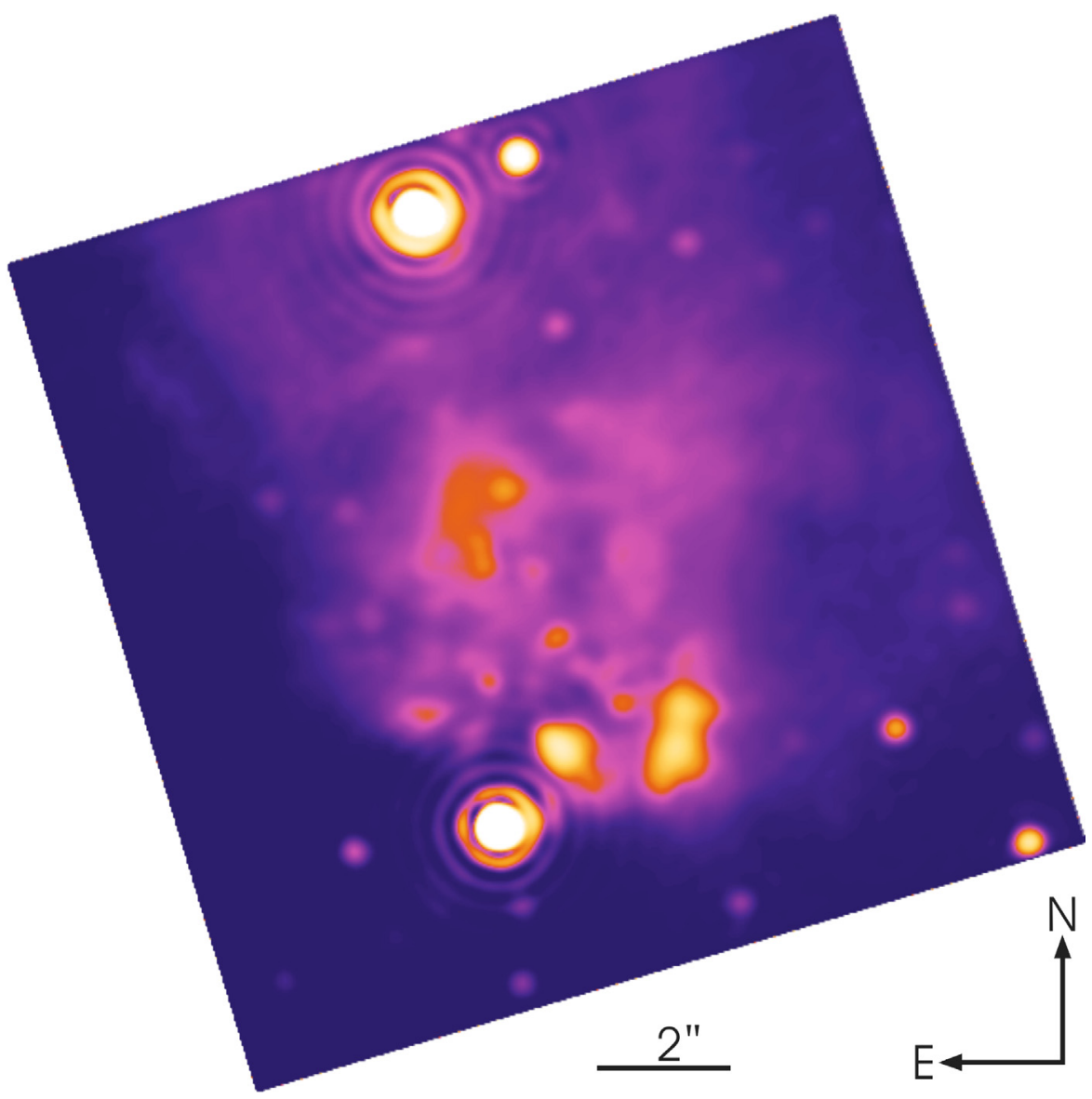

a) MMT speckle image (334 mas resolution, 2004-12-20).

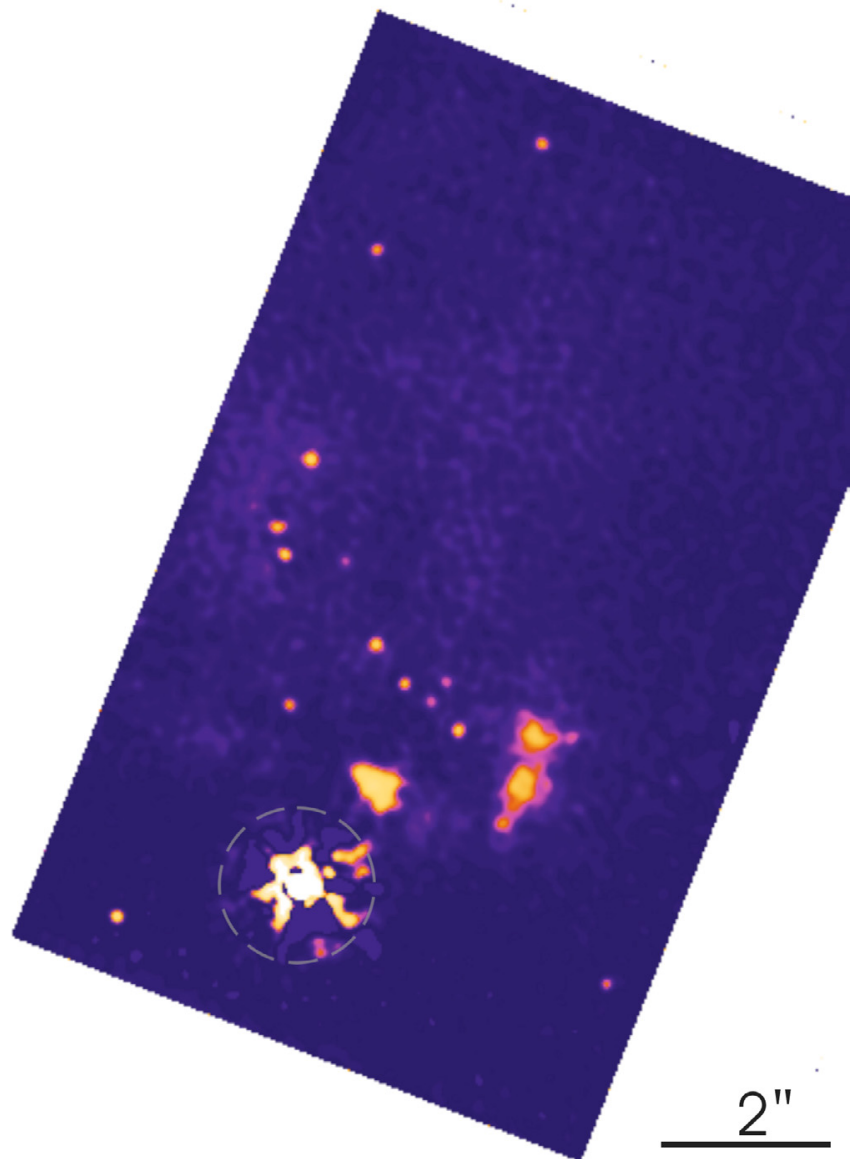

b) BTA speckle image (146 mas resolution, 2002-09-24).
Fig. 1. Bispectrum speckle images ( $K^{\prime}$-band) reconstructed from data taken with a) the $6.5 \mathrm{~m} \mathrm{MMT}$ and b) the $6 \mathrm{~m}$ BTA telescope. To show the weak emission features, the intensity of IRS1 was clipped to $2 \%$ of the total flux. Within the high-resolution image b), speckle-noise artifacts appear around IRS1 (marked with a circle). These weak features represent small distortions of the point-spreadfunction (PSF) on the 1\%-level and do not influence the reliability of the identification of point sources within the image. The absolute coordinates of IRS1 are $\alpha=23^{\mathrm{h}} 13^{\mathrm{m}} 45^{\mathrm{s}} .35$ and $\delta=+61^{\circ} 28^{\prime} 10^{\prime} 84(\mathrm{~J} 2000$, determined from 2MASS, accuracy $\sim 0$ '. $^{\prime}$ ). 


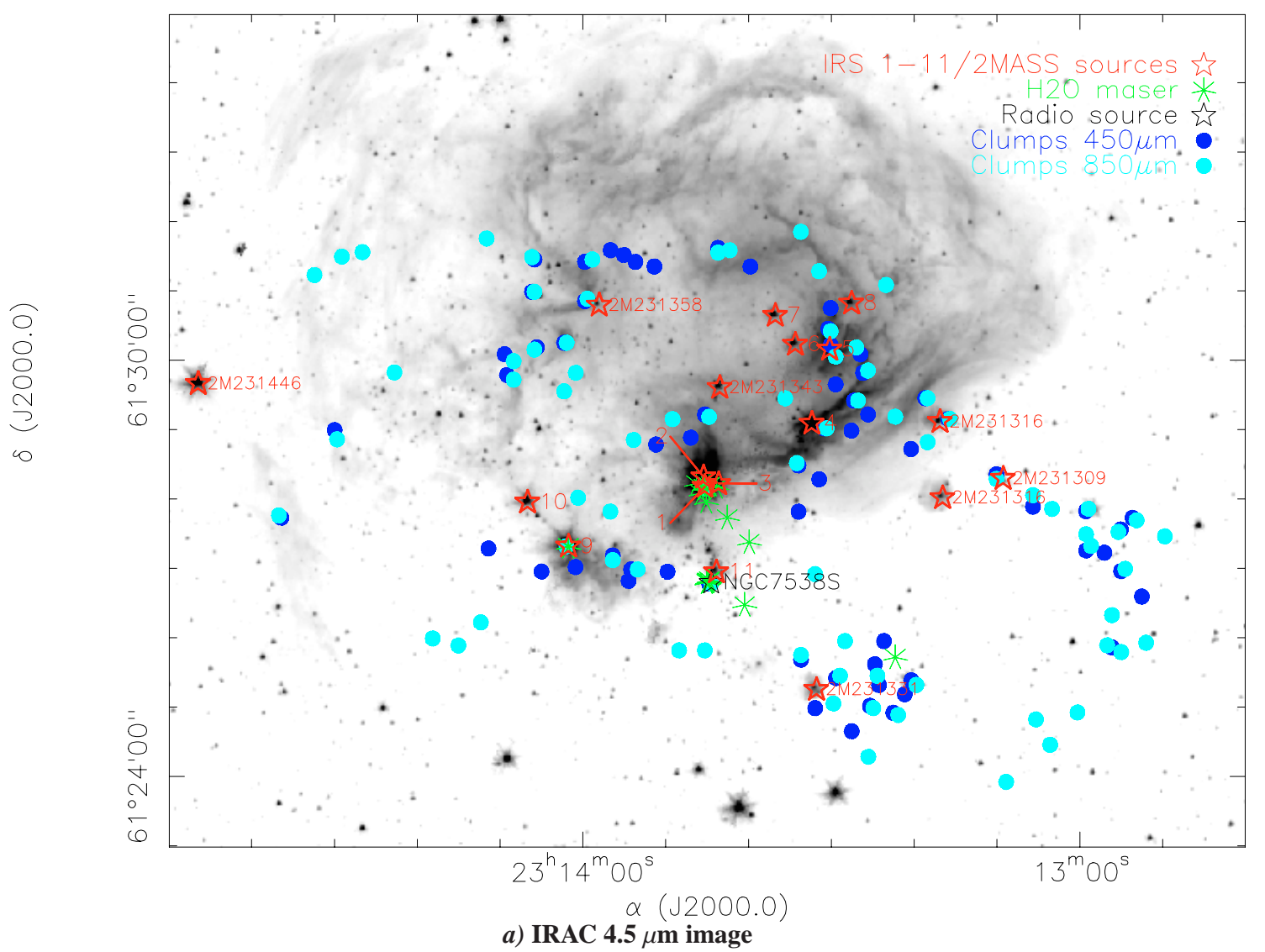

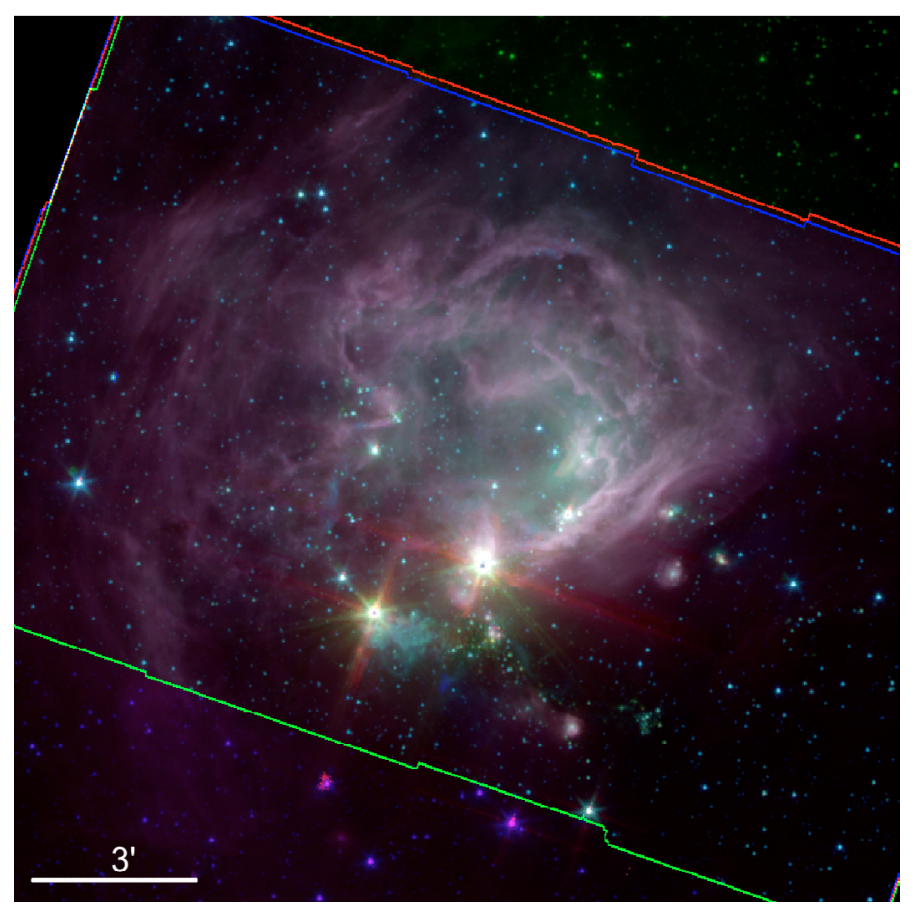

b) IRAC color composite: $3.6 / 4.5 / 5.8 \mu \mathrm{m}$

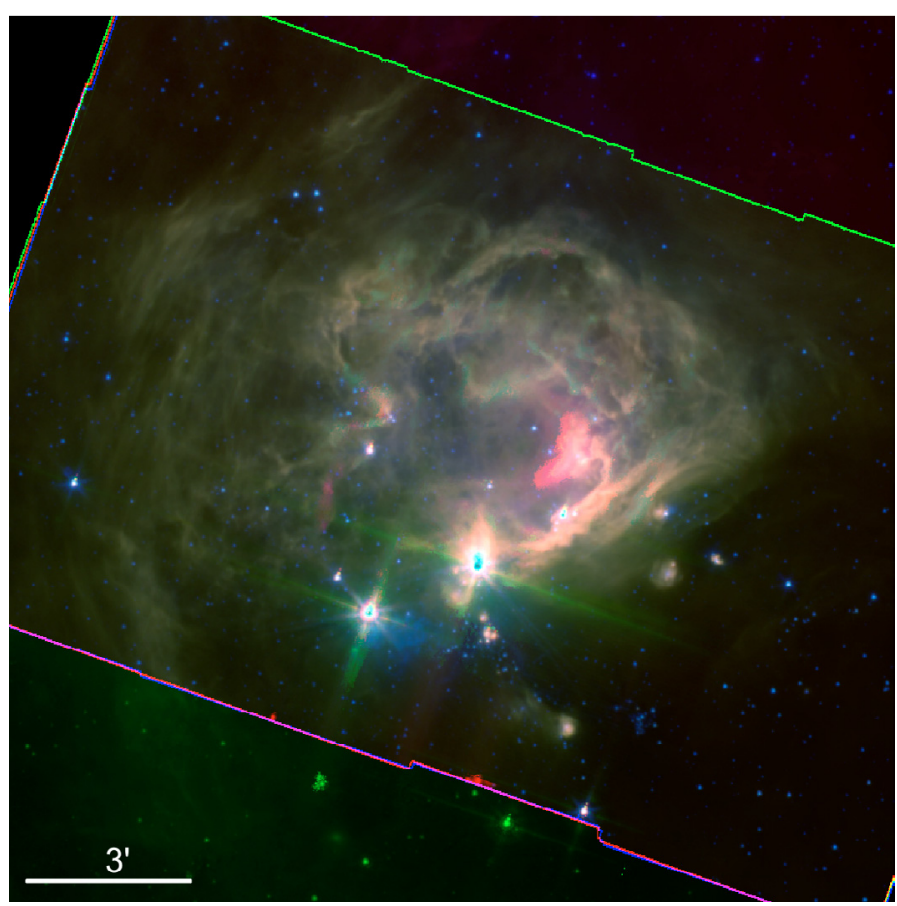

c) IRAC color composite: $4.5 / 5.8 / 8.0 \mu \mathrm{m}$

Fig. 2. a) shows the Spitzer/IRAC $4.5 \mu \mathrm{m}$ image with the position of the infrared sources (IRS1 to 11 and $2 \mathrm{MASS}$ sources) and $\mathrm{H}_{2} \mathrm{O}$ masers marked. Furthermore, the position of the sub-millimeter ( $450 \mu \mathrm{m}$ and $850 \mu \mathrm{m})$ clumps reported by Reid \& Wilson (2005) are shown. The position of the 2MASS sources 2MASS 23135808+6130484, 2MASS 23131660+6128017, 2MASS 23134351+6129372, 2MASS 23144651+6129397, 2MASS 23131691+6129076, 2MASS 23130929+6128184, and 2MASS 23133184+6125161 are labeled explicitly. b), c) show color-composites produced with two triplets of the four IRAC bands. The intensity of each image was scaled logarithmically. North is up and east to the left. 


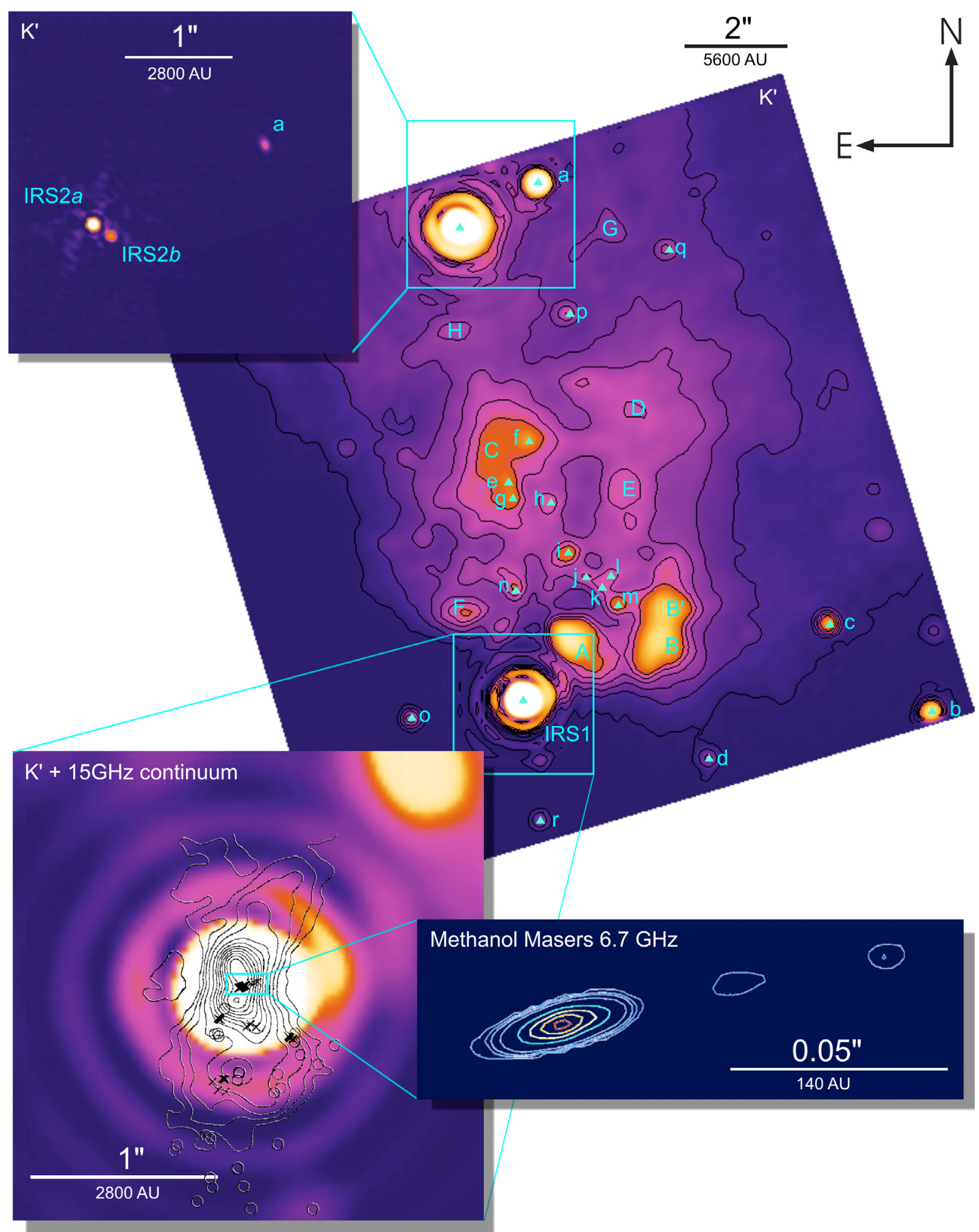

Fig. 3. Bispectrum speckle image with identified point sources (triangles) atop marked. The astrometry for the point-sources was performed using the high-resolution BTA image, whereas the image shown was reconstructed from MMT data. The contours trace $0.25 \%, 0.5 \%, 0.75 \%$, $1.0 \%, 1.25 \%$, and $1.5 \%$ of the peak intensity. The inset on the upper left shows a reconstruction of the vicinity of IRS2 using a resolution of 80 mas (BTA data). In the lower left, IRS1 is shown using a different color table, emphasizing the elongation of the IRS1 Airy disk (MMT data) overplotted with the $15 \mathrm{GHz}$ radio continuum (the contours show $-1,1,2.5,5,10,20, \ldots, 90 \%$ of the peak flux) and the position of the OH (circles) and methanol (crosses) masers (image from Hutawarakorn \& Cohen 2003; using data from Gaume et al. 1995). In the lower right we show the integrated brightness of the methanol masers as presented by Pestalozzi et al. (2004, contour levels of 1, 3, 5, 10, 30, 50, 70, and 90\% of the peak flux density are shown). 


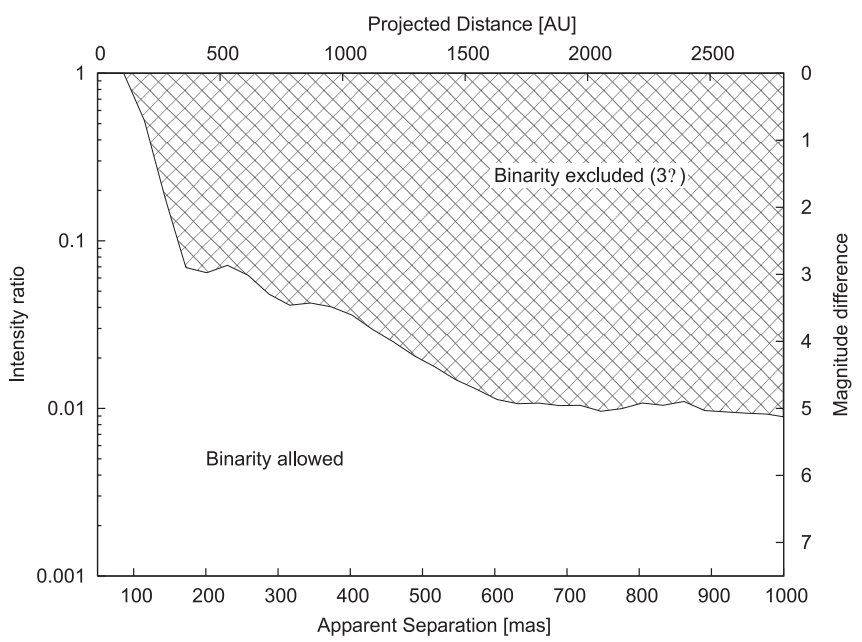

Fig. 4. By measuring the speckle noise around the PSF of IRS1, we can rule out binarity of IRS1 on a $3 \sigma$-level as a function of apparent separation and intensity ratio.

conical (fan-shaped) region with a $90^{\circ}$ opening angle extending from IRS1 towards the northwest.

A careful examination of the power spectrum of IRS1 has shown that the detected asymmetry of IRS 1 is not caused by a companion, but seems to represent diffuse emission. Therefore, we can rule out a close binary system of similar-brightness components down to the diffraction limit of $\sim 70$ mas. For the case of a binary system with components of significantly different brightnesses, we can put upper limits on the brightness of the hypothetical companion as a function of the projected binary separation (see Fig. 4).

The PA of the elongation of the Airy disk is similar to the PA of $K^{\prime}$ blobs $A, B$, and $B^{\prime}$. Another strong feature $(C)$ can be seen towards $\mathrm{PA} \sim 0^{\circ}$. The blobs seem to be connected by a bridge of diffuse emission extending from feature $B$ to $C$. Overall, the diffuse emission seems to form a fan-shaped region which is extending from IRS1 towards the northwest with an opening angle of nearly $90^{\circ}$. We identified some further features and list their position angles and separations in Table 1 . The directions, which were reported for various outflow tracers, are also listed in this table and illustrated in Fig. 5.

Our features $A, B+B^{\prime}$, and $C$ appear to coincide with the features $A, B$, and $C$ identified by Alvarez et al. (2004). A comparison suggests that features $A+B+B^{\prime}, C, D$ correspond to the peaks $1 W, 1 N$, and $1 N W$ in Tamura et al. (1991).

\subsubsection{Binarity of NGC 7538 IRS2}

IRS2 appears resolved as a close binary system. Using an image reconstructed from BTA data with a spatial resolution of 80 mas (see inset in the upper-left corner of Fig. 3), we determined the separation to be 195 mas and found a PA of $-123^{\circ}$ for the 1. 9 fainter companion (2002-09-24). We designate the brighter component in the $K^{\prime}$-band as IRS $2 a$ and the fainter as IRS $2 b$.

\subsubsection{Detection of fainter cluster members}

Besides IRS 1 and IRS $2 a / b$, we were able to identify 18 additional fainter point-like sources $(a-r)$ within the BTA image, whose positions and $K^{\prime}$-band magnitudes are listed in Table 2.

In order to test whether these sources are physically related to NGC 7538, one can compare the stellar number density for the brightness range $11 \mathrm{~m}^{\mathrm{m}} 0$ to $12^{\mathrm{m}} 0$ in our speckle image, $N_{\text {Speckle }}=18 / 128 \operatorname{arcsec}^{2} \approx 2.1 \times 10^{6} / \mathrm{deg}^{2}$, with the number expected from the cumulative $K$-band luminosity function (KLF) of the NGC 7538 field $^{2} N_{\text {field }} \approx 1.8 \times 10^{3} / \mathrm{deg}^{2}$. Although these number densities were obtained with different spatial resolution, the clear over-density of stars in our speckle image is significant and we conclude that most of the detected stars are likely members of the NGC 7538 star forming region. When using the KLF for the IRS 1-3 region instead of the whole NGC 7538 field, the stellar over-density in our speckle image becomes even more evident $\left(N_{\text {IRS1-3 cluster }} \approx 1.4 \times 10^{3} / \mathrm{deg}^{2}\right)$. Since these stars are about 5 to 6 mag fainter than IRS1, they are likely to be part of the associated intermediate mass stellar population.

The arrangement of the stars within the fan-shaped nebula does not appear to be random, but follows the S-structure of the diffuse blobs (see Fig. 3). Most remarkable, more than half of the stars seem to be aligned in a chain reaching from feature $B$ to $C$ $\left(\mathrm{PA} \sim 45^{\circ}\right)$. Within the diffuse blobs close to IRS1 $\left(A, B, B^{\prime}\right)$, no stars were found, whereas embedded in blob $C$, three stars could be detected.

\subsection{Spitzer/IRAC: morphology at large spatial scales}

Imaging of NGC 7538 at optical wavelengths showed that diffuse emission can mainly be found in the vicinity of IRS5 (Lynds \& O'Neil 1986). At near-infrared (NIR) wavelengths (Ojha et al. 2004), a diffuse structure can be found extending from the IRS1-3 cluster towards the northwest with the strongest emission around IRS5.

The Spitzer/IRAC images reveal a more complex, bubblelike structure (see Figs. 2b, c), whose western border is formed by a pronounced ridge-like filament connecting IRS1-3 with IRS4 and reaching up to IRS5 (see Fig. 2a). At the western border of the bubble a wide conical structure is located, with a vertex on 2MASS 23135808+6130484. Another conical structure can be detected close to the northern border of the bubble. Several other outflow structures can be found in the IRAC image; most noteworthy, the unidirectional reflection nebula around 2MASS 23144651+6129397, 2MASS 23131691+6129076, and 2MASS 23130929+6128184 (see Fig. 2a). The sources 2MASS 23131660+6128017 and 2MASS 23133184+6125161 appear to be embedded in a shelllike cloud.

Besides the position of the strongest near-infrared sources, Fig. 2 shows also the position of the submillimeter $(450 \mu \mathrm{m}$, $850 \mu \mathrm{m})$ clumps reported by Reid \& Wilson (2005). These clumps trace the filaments and knots of the bubble, which can be seen in the IRAC images, very well. Besides this, the submillimeter clumps suggest another bubble-like structure to the southwest of IRS4 (see also the images in Reid \& Wilson 2005). This bubble seems to be invisible at nearand mid-infrared wavelengths, although several NIR sources are located on its border (2MASS 23130929+6128184, 2MASS 23133184+6125161).

As already pointed out by Reid \& Wilson (2005), it is interesting to compare the position of the detected $\mathrm{H}_{2} \mathrm{O}$ masers with the position of the centers of high-mass star formation in the region and to find agreement in many cases (IRS1-3, IRS9, NGC 7538S). However, as can be seen in Fig. 2, for four locations of $\mathrm{H}_{2} \mathrm{O}$ masers, no MIR counterpart can be found in

\footnotetext{
2 The $K$-band luminosity function by Balog et al. (2004) for the whole NGC 7538 region, corrected with the on-cluster KLF, and cumulated for the magnitude range 11 . 0 to 12 . 0 was used.
} 
Table 1. NGC 7538 IRS1 outflow directions reported for various tracers.

\begin{tabular}{|c|c|c|c|c|c|c|c|}
\hline Tracer & Structure & $\begin{array}{c}\text { Beam size } \\
{\left[{ }^{\prime \prime}\right]}\end{array}$ & $\begin{array}{c}\text { Scale } \\
{\left[{ }^{\prime \prime}\right]}\end{array}$ & $\begin{array}{c}\text { PA of } \\
\text { outflow } \\
\text { direction }\left[^{\circ}\right]\end{array}$ & $\begin{array}{r}\text { Dynamical } \\
\text { age }^{f} \\
{\left[10^{3} \mathrm{yr}\right]}\end{array}$ & Ref. & $\begin{array}{l}\text { Comments } \\
\text { (velocities in }\left[\mathrm{km} \mathrm{s}^{-1}\right] \text { ) }\end{array}$ \\
\hline Methanol masers & & $0.03^{c}$ & & $+19^{a, d}$ & - & {$[8]$} & see Fig. 3 \\
\hline $1.0 \mathrm{~cm}$ cont. & inner core $\lesssim 0.0^{\prime} 5$ & 0.13 & 0.4 & $+0^{a}$ & $<0.03$ & [9] & $t_{\mathrm{e}}=0.15 \mathrm{yr}^{e}$ \\
\hline $1.3 \mathrm{~cm}$ cont. & inner core $\lesssim 0^{\prime} 4$ & 0.11 & 0.4 & $+0^{a}$ & $<0.02$ & [5] & \\
\hline $6.0 \mathrm{~cm}$ cont. & inner core $\lesssim 0 . ' 75$ & 0.35 & 0.4 & +0 & $<0.04$ & [1] & \\
\hline $1.0 \mathrm{~cm}$ cont. & outer core $\gtrsim 0 .{ }^{\prime} 5$ & 0.13 & 1.0 & $-25^{a}$ & $>0.03$ & [9] & $t_{\mathrm{e}}=0.3 \mathrm{yr}^{e}$ \\
\hline $1.3 \mathrm{~cm}$ cont. & outer core $\gtrsim 0 .{ }^{\prime} 4$ & 0.11 & 1.0 & $-25^{a}$ & $>0.02$ & [5] & \\
\hline $6.0 \mathrm{~cm}$ cont. & outer core $\gtrsim 0.75$ & 0.35 & 1.0 & $-15 \ldots-20$ & $>0.04$ & [1] & \\
\hline $6.0 \mathrm{~cm}$ cont. & outer core $\gtrsim 0 .{ }^{\prime} 5$ & 0.4 & 1.0 & $-25^{a}$ & $>0.03$ & [9] & \\
\hline MIR $11.7 \mu \mathrm{m}$ & IRS1 Elongation & 0.43 & 3 & -45 & 0.16 & [10] & see Fig. 6k \\
\hline MIR $18.3 \mu \mathrm{m}$ & IRS1 Elongation & 0.54 & 4 & -45 & 0.16 & [10] & see Fig. 61 \\
\hline NIR $K^{\prime}$-band & IRS1 Elongation & 0.3 & 0.6 & -78 & 0.03 & - & see Fig. 3 \\
\hline NIR $K^{\prime}$-band & feature A & 0.3 & 1.6 & -65 & 0.08 & - & see Fig. 3 \\
\hline NIR $K^{\prime}$-band & feature $\mathrm{F}$ & 0.3 & 2.1 & -33 & 0.11 & - & see Fig. 3 \\
\hline NIR $K^{\prime}$-band & feature B & 0.3 & 3.0 & -39 & 0.16 & - & see Fig. 3 \\
\hline NIR $K^{\prime}$-band & feature $B^{\prime}$ & 0.3 & 3.3 & -57 & 0.18 & - & see Fig. 3 \\
\hline NIR $K^{\prime}$-band & feature $\mathrm{C}$ & 0.3 & 4.8 & +6 & 0.25 & - & see Fig. 3 \\
\hline NIR $K^{\prime}$-band & feature $\mathrm{E}$ & 0.3 & 6.2 & -20 & 0.33 & - & see Fig. 3 \\
\hline NIR $K^{\prime}$-band & feature D & 0.3 & 7.4 & +10 & 0.39 & - & see Fig. 3 \\
\hline NIR $K^{\prime}$-band & eastern wall & 0.3 & - & +25 & - & - & see Fig. 3 \\
\hline NIR $K^{\prime}$-band & western wall & 0.3 & - & -65 & - & - & see Fig. 3 \\
\hline$[\mathrm{Fe} \mathrm{II}] 1.65 \mu \mathrm{m}$ & & 1 & $15^{a}$ & $\mathrm{~N}-\mathrm{S}^{a}$ & 0.8 & [7] & around IRS2; see Fig. 6 i \\
\hline $\mathrm{H}_{2}$ & northern bow & & $30^{a}$ & $\mathrm{~N}-\mathrm{S}$ & 1.5 & [6] & \\
\hline $\mathrm{H}_{2}$ & & 1 & $27^{a}$ & $-25^{a}$ & 1.4 & [7] & shell-like structure; see Fig. 6i \\
\hline $\mathrm{H}_{2}$ & southern bow & & $45^{a}$ & $155^{a}$ & 2.3 & {$[6]$} & see Fig. $6 \mathrm{~h}$ \\
\hline IRAC bands & southern bow & 1 & 40 & 145 & 2.2 & - & see Fig. 6a...e \\
\hline $\mathrm{CO}$ & low velocities & 7 & $5^{a, b}$ & E-W & 0.9 & [3] & $-11<\tilde{V}_{\mathrm{b}}<-6 ; 2<\tilde{V}_{\mathrm{r}}<9$; see Fig. $6 \mathrm{~g}$ \\
\hline $\mathrm{CO}$ & high velocities & 7 & $15^{b}$ & -35 & 15 & {$[3]$} & $-17<\tilde{V}_{\mathrm{b}}<-11 ; 9<\tilde{V}_{\mathrm{r}}<15 ;$ see Fig. $6 \mathrm{~g}$ \\
\hline $\mathrm{CO}$ & & 34 & $18^{b}$ & $-50^{a}$ & 15 & {$[2]$} & $-24<\tilde{V}_{\mathrm{b}}<-8 ; 9<\tilde{V}_{\mathrm{r}}<22$ \\
\hline $\mathrm{CO}$ & & 16 & $13^{a, b}$ & -40 & 14 & [4] & $-14<\tilde{V}_{\mathrm{b}}<-9 ; 11<\tilde{V}_{\mathrm{r}}<16$; see Fig. 6f \\
\hline $\mathrm{CO}$ & & 45 & $12^{a, b}$ & $-50^{a}$ & 10 & [3] & $-24<\tilde{V}_{\mathrm{b}}<-8 ; 9<\tilde{V}_{\mathrm{r}}<22$ \\
\hline
\end{tabular}

Note $-\tilde{V}_{\mathrm{r}}$ and $\tilde{V}_{\mathrm{b}}$ are measured relative to the velocity of methanol maser feature A $\left(\tilde{V}=V-56.25 \mathrm{~km} \mathrm{~s}^{-1}\right){ }^{a}$ Estimated from figures presented within the reference paper; therefore, with limited accuracy; ${ }^{b}$ the half-separation between the red- and blue-shifted CO lobe is given; ${ }^{c}$ for VLBI observations, we give the estimated error on the absolute position of the measured maser spots; ${ }^{d}$ the expected outflow direction is given; i.e., perpendicular to the measured orientation of maser feature $\mathrm{A} ;{ }^{e}$ electron recombination time given in the reference paper; ${ }^{f}$ assuming an outflow velocity of $250 \mathrm{~km} \mathrm{~s}^{-1}$, which was measured by Gaume et al. (1995) within the H66 $\alpha$ recombination line. For the CO emission, we also use the measured $\mathrm{CO}$ outflow velocity and provide the corresponding dynamical age in brackets. Since all velocities are measured along LOS, this timescale can only provide upper limits.

References: [1] Campbell (1984); [2] Fischer et al. (1985); [3] Scoville et al. (1986); [4] Kameya et al. (1989); [5] Gaume et al. (1995); [6] Davis et al. (1998); [7] Bloomer et al. (1998); [8] Minier et al. (2000); [9] Franco-Hernández \& Rodríguez (2004); [10] De Buizer \& Minier (2005).

the IRAC images (the detection limits for point sources in the four IRAC bands are roughly $3.6,5.3,31$, and $34 \mu \mathrm{Jy}$ for the IRAC bands at 3.6, 4.5, 5.8, and $8 \mu \mathrm{m}$ assuming medium sky background).

\section{Discussion}

\subsection{Nature of the observed $K^{\prime}$-band emission}

In the wavelength range of the $K^{\prime}$-band filter $\left(\lambda_{0}=2.12 \mu \mathrm{m}\right.$, $\Delta \lambda=0.21 \mu \mathrm{m})$, we record not only continuum radiation (e.g. scattered light, thermal dust emission, stellar continuum emission), but also line emission (e.g. $\mathrm{H}_{2}$ ). However, both Bloomer et al. (1998, see Fig. 6) and Davis et al. (1998) did not detect significant amounts of $\mathrm{H}_{2}$ emission around IRS1. Furthermore, deep optical imaging by Elsaesser et al. (1982) and Campbell \& Persson (1988) reveal a weak optical source offset 2 '. 2 north of the radio source IRS1. The latter authors argue that the strong extinction $\left(A_{\mathrm{V}}=13\right)$ derived for IRS1 makes it highly unlikely that this optical emission is connected to IRS1 itself but that it most likely represents scattered light. The measured offset suggests that the faint optical source should be associated with blobs $A$ and $B$ in our images, making scattering the most likely radiation mechanism for the detected $K^{\prime}$-band emission. This conclusion is also supported by polarization measurements (Dyck \& Lonsdale 1979) which show a strong polarization of the $2 \mu \mathrm{m}$ emission, tracing either scattered light or light transmitted through aligned grains. Henceforth we presume continuum to be the most important contributor to the detected emission.

\subsection{Methanol maser feature A: protostellar disk or outflow?}

We note that the 2 MASS position of IRS1 $\left(\alpha=23^{\mathrm{h}} 13^{\mathrm{m}} 45^{\mathrm{s}} .35\right.$, $\left.\delta=+61^{\circ} 28^{\prime} 10^{\prime} .8, \mathrm{~J} 2000\right)$ and the position of the methanol maser feature A $\left(\alpha=23^{\mathrm{h}} 13^{\mathrm{m}} 45^{\mathrm{s}} .364, \delta=+61^{\circ} 28^{\prime} 10^{\prime} .55, \mathrm{~J} 2000\right)$ reported by Minier et al. (2000) coincide within the errors ${ }^{3}$. Therefore, the methanol masers and the outflow driving source

3 The astrometric accuracy of the 2MASS catalogue was reported to be $\sim 0$ '.15 (see http://ipac. caltech. edu/2mass/releases/ allsky/doc/expl-sup.html). 
Fig. 5. Illustration showing the outflow directions in the various tracers. The CO contours by Kameya et al. (1989, red and blue) are overlaid on the $\mathrm{H}_{2}$ map (greyscale) by Davis et al. (1998). The orientation of the conjectural methanol maser disk (green), the fan-shaped structure detected in our $K^{\prime}$-band image (orange), and the averaged direction of $\mathrm{H}_{2}$ (red arcs) are shown schematically. The arrows indicate the direction prependicular to the alignment of the methanol masers (green), the orientation of the inner $\left(<0.5^{\prime \prime}\right)$ and outer $\left(>0.5^{\prime \prime}\right)$ core detected in the $1.0,1.3$, and $6.0 \mathrm{~cm}$ radio continuum (white), and the direction along which the IRS1 Airy disk was found to be elongated (MIR: De Buizer \& Minier 2005; NIR: this paper).

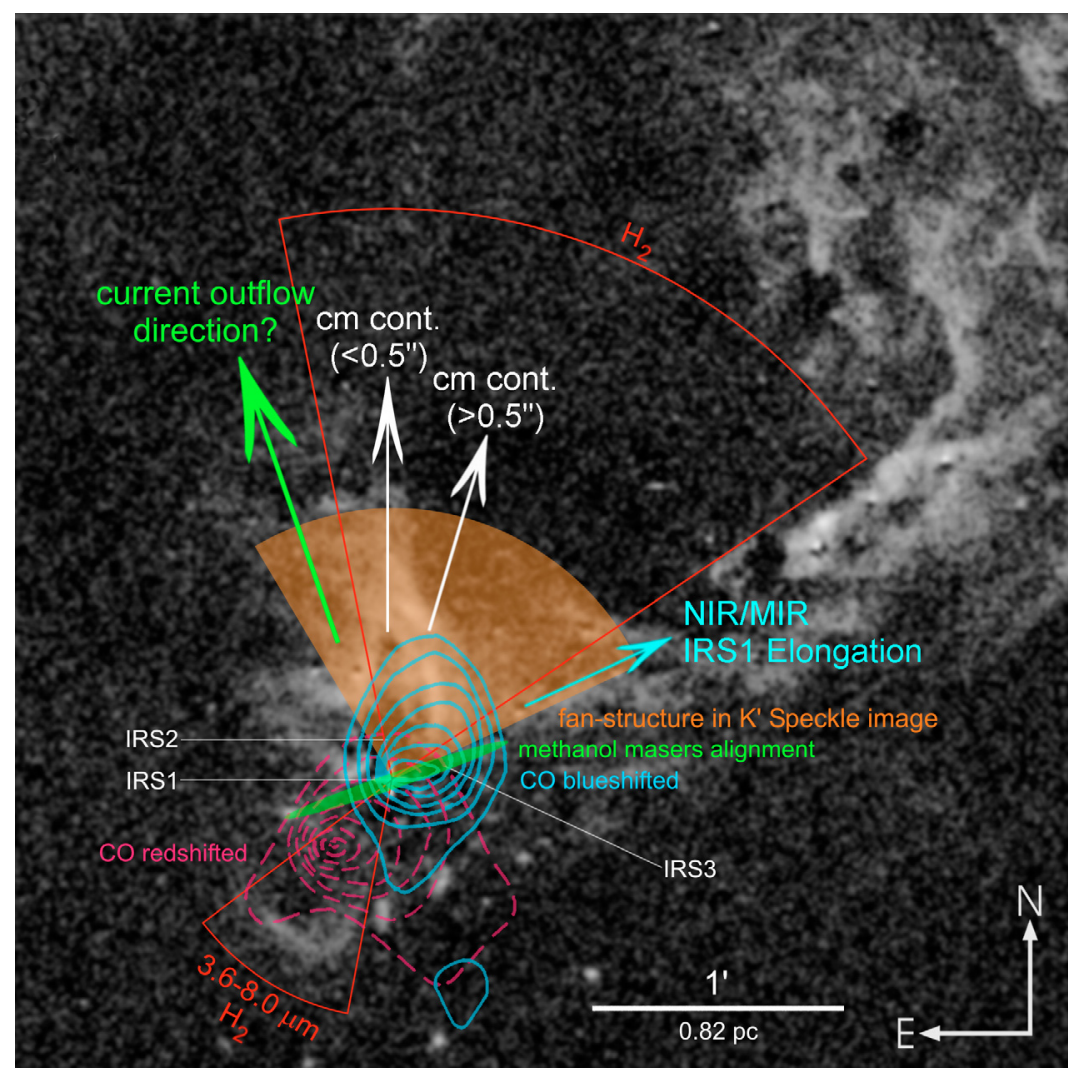

Table 2. Point sources identified in our speckle images. For details about IRS1 and the binary system IRS2a/b, we refer to the text. We identify components $a$ to $f$ with the stars already discovered in the image by Alvarez et al. (2004).

\begin{tabular}{|c|c|c|c|c|}
\hline Name & $\mathrm{RA}(\mathrm{J} 2000)^{a}$ & $\operatorname{Dec}(\mathrm{J} 2000)^{a}$ & $K^{\prime}$ Magnitude $^{b}$ & Comment \\
\hline $\mathrm{a}$ & $23^{\mathrm{h}} 13^{\mathrm{m}} 45^{\mathrm{s}} .33$ & $61^{\circ} 28^{\prime} 21^{\prime \prime} .02$ & $10^{\mathrm{m}} 94$ & \multirow{18}{*}{$\begin{array}{l}\text { embedded in feature } C \\
\text { embedded in feature } C \\
\text { embedded in feature } C\end{array}$} \\
\hline b & $44^{\$} .81$ & $100^{\prime} 63$ & $11^{\mathrm{m}} \cdot 29$ & \\
\hline $\mathrm{c}$ & 44.95 & $12^{\prime \prime} .33$ & $11^{\mathrm{m}} \cdot 46$ & \\
\hline d & $45^{5} .11$ & 09.'69 & $11^{\mathrm{m}} \cdot 73$ & \\
\hline $\mathrm{e}$ & $45^{\text {s.37 }}$ & $15^{\prime \prime} 12$ & 11.77 & \\
\hline $\mathrm{f}$ & $45^{5} .34$ & $15^{\prime \prime} 93$ & 11.73 & \\
\hline g & $45^{\varsigma} .36$ & $14^{\prime \prime} 80$ & $11^{\mathrm{m}} \cdot 73$ & \\
\hline $\mathrm{h}$ & $45^{\varsigma} .31$ & $14^{\prime \prime} 72$ & 11.86 & \\
\hline $\mathrm{i}$ & $45^{5} .29$ & $133^{\prime \prime} 74$ & $11^{\mathrm{m}} \cdot 71$ & \\
\hline $\mathrm{j}$ & $45^{5} .27$ & $133^{\prime \prime} 25$ & $11^{\mathrm{m}} \cdot 73$ & \\
\hline $\mathrm{k}$ & $45^{5} .25$ & $133^{\prime \prime} 05$ & $11^{\mathrm{m}} \cdot 81$ & \\
\hline 1 & $45^{5} .24$ & 13". 28 & $11 . \mathrm{m} 83$ & \\
\hline $\mathrm{m}$ & $45^{\varsigma} .22$ & $12^{\prime \prime} 71$ & $11 . \mathrm{m} \cdot 71$ & \\
\hline $\mathrm{n}$ & $45^{5} .36$ & $12^{\prime \prime} .99$ & $11^{\mathrm{m}} \cdot 75$ & \\
\hline o & 45.50 & $10 ! 50$ & $11^{\mathrm{m}} \cdot 60$ & \\
\hline $\mathrm{p}$ & $45^{5} .29$ & $18^{\prime \prime} .42$ & $11^{\mathrm{m}} \cdot 73$ & \\
\hline$q$ & 45.16 & $19 ! 69$ & $11^{\mathrm{m}} \cdot 77$ & \\
\hline $\mathrm{r}$ & $45^{5} .33$ & $08^{\prime \prime} .48$ & $11^{\mathrm{m}} \cdot 71$ & \\
\hline
\end{tabular}

${ }^{a}$ For the astrometry, the relative errors are of the order of 0 ' 1 . The absolute calibration using the reference position of IRS1 in 2 MASS introduces further errors $(0,2) ;{ }^{b}$ the photometry was done relative to IRS1 with an uncertainty of 0.3 . For the conversion to absolute photometry, we assumed a IRS1 magnitude of 8.9 (Ojha et al. 2004).

are likely causually connected, however a random coincidental alignment cannot be ruled out.

Since methanol masers can trace both protostellar disks and outflows, it is not a priori clear how the linear alignment of the methanol maser feature A and the observed velocity gradient should be interpreted. For IRS1, both claims have been made (Pestalozzi et al. 2004; De Buizer \& Minier 2005). However, detailed modeling has provided strong quantitative support for the disk interpretation but is still missing for the outflow interpretation. Furthermore, a study by Pestallozi et al. (in prep.) suggests that simple outflow geometries cannot explain the observed properties of feature A.

A major difference between these two scenarios is the orientation of the disk associated with the outflow driving source: Whereas in the disk scenario the methanol masers are lined up within the disk plane (PA $\sim 62^{\circ}$ ), the outflow scenario suggests an orientation of the disk plane perpendicular to the maser alignment $\left(\mathrm{PA} \sim+28^{\circ}\right)$. The observed asymmetry in our 


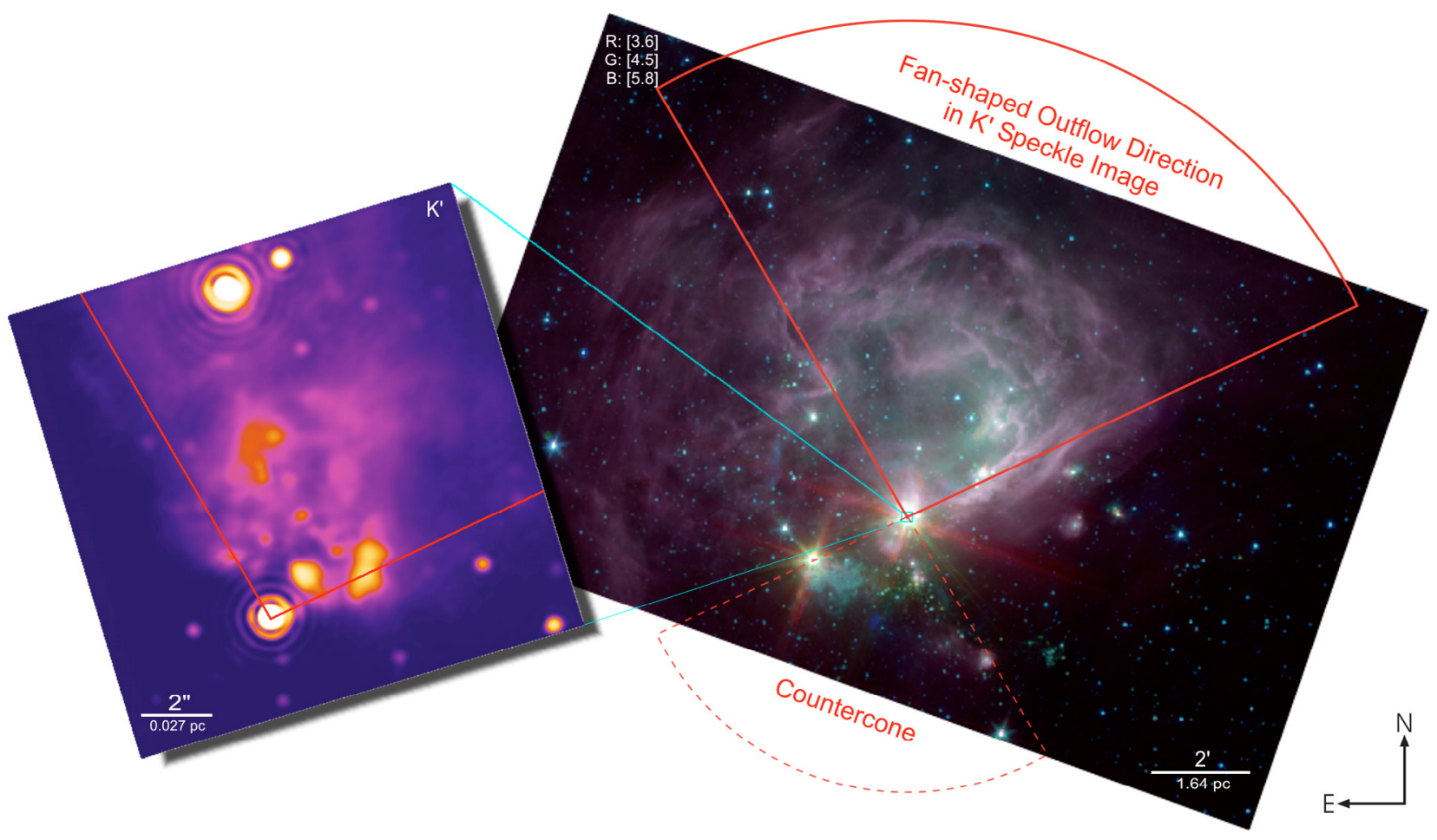

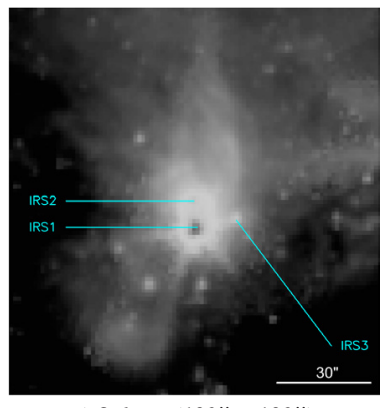

a) $3.6 \mu \mathrm{m}\left(120^{\prime \prime} \times 120^{\prime \prime}\right)$
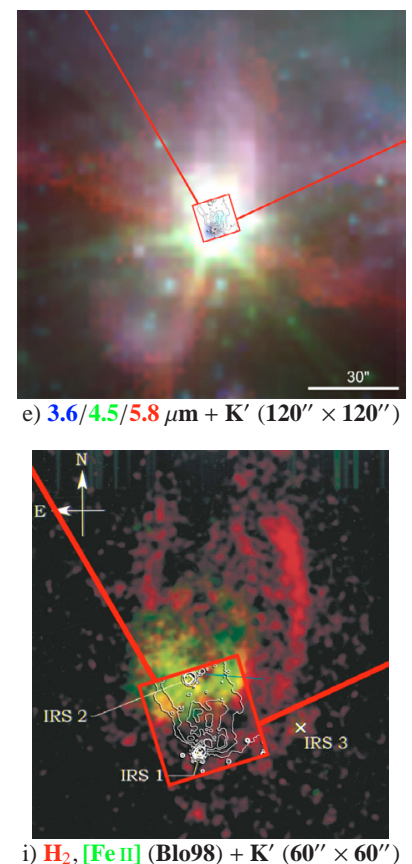

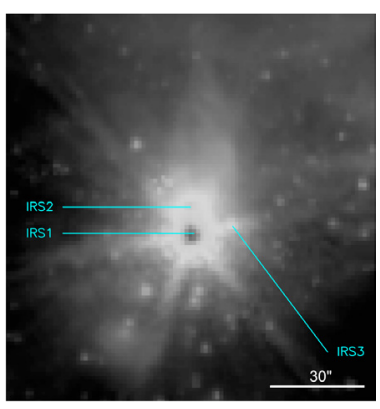

b) $4.5 \mu \mathrm{m}\left(120^{\prime \prime} \times 120^{\prime \prime}\right)$

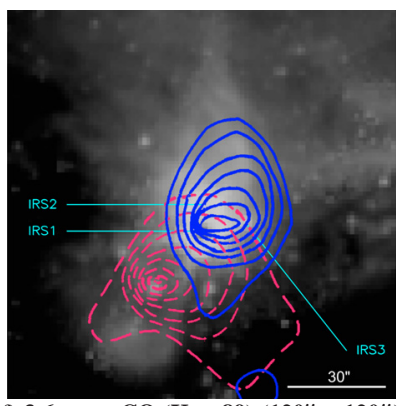

f) $3.6 \mu \mathrm{m}+\mathrm{CO}(\mathrm{Kam89})\left(120^{\prime \prime} \times 120^{\prime \prime}\right)$

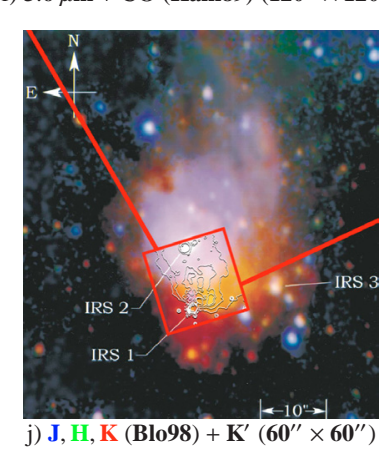

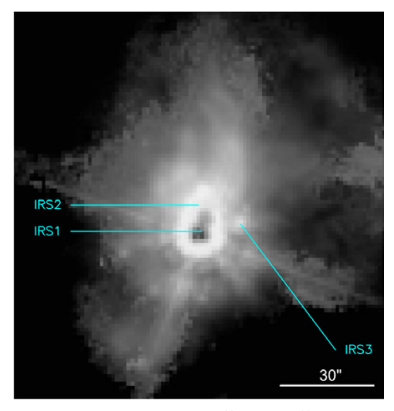

c) $5.8 \mu \mathrm{m}\left(120^{\prime \prime} \times 120^{\prime \prime}\right)$
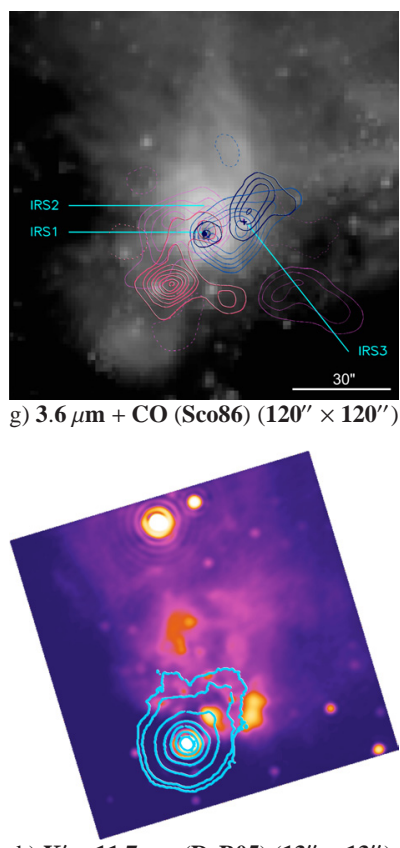

k) $\mathrm{K}^{\prime}+11.7 \mu \mathrm{m}(\mathrm{DeB05})\left(13^{\prime \prime} \times 13^{\prime \prime}\right) \quad$ 1) $\mathrm{K}^{\prime}+18.3 \mu \mathrm{m}(\mathrm{DeB05})\left(\mathbf{1 3}^{\prime \prime} \times 13^{\prime \prime}\right)$

Fig. 6. Mosaic showing the IRS1-3 cluster at various wavelengths. Beside the speckle $K^{\prime}$-band image (also marked as red box) and IRAC images, data from Scoville et al. (1986, CO), Kameya et al. (1989, CO), Bloomer et al. (1998, J, H, K, $\mathrm{H}_{2}$, [Fe II]), Davis et al. (1998, $\mathrm{H}_{2}$ ), and De Buizer $\&$ Minier $(2005,11.7 \mu \mathrm{m}, 18.3 \mu \mathrm{m})$ was incorporated. 
NIR speckle images, as well as the elongation of the emission observed in the 11.7 and $18.3 \mu \mathrm{m}$ images by De Buizer \& Minier (2005), can be explained within both scenarios:

Scenario A: if maser feature A traces an outflow cavity, the detected asymmetry might simply reflect the innermost walls of this cavity (oriented northwest), whereas the southeastern cavity of a presumably bipolar outflow might be hidden due to inclination effects.

Scenario B: alternatively, if the masers trace an edge-on circumstellar disk, the asymmetry of the infrared emission could trace the western wall of an outflow cavity with a wide half-opening angle. The asymmetry cannot be attributed to the disk itself because the detection of stellar radiation scattered off the disk surface at such a large distance is highly unlikely.

For completeness, we also mention the interpretation by Kameya et al. (1989), who attributed the change between the direction observed in the $\mathrm{UCH}$ II region $\left(\mathrm{PA} 0^{\circ}\right.$ ) and the highvelocity $\mathrm{CO}$ flow $\left(\mathrm{PA}-60^{\circ}\right)$ to flow deflection, either by largescale magnetic fields or due to density gradients.

We proceed now to discuss both scenarios within an outflowcavity model (Sect. 4.3) and a precessing jet model (Sect. 4.4), incorporating the large amounts of evidence collected by various authors over the last three decades.

\subsection{Scenario A: outflow cavity model}

Since the intensity of the diffuse emission in our images seems to decrease with distance from IRS1 and the vertex of the fanshaped region appears centered on IRS1, we cannot support the hypothesis by Bloomer et al. (1998), who identified IRS2 as the likely source of the diffuse NIR emission. Instead, the observed fan-shaped region can be interpreted as a cavity that was formed by outflow activity from IRS1. Because the walls of the fan-shaped structure are well-defined, we can measure the opening angle of the proposed outflow cavity from the eastern wall (PA $25^{\circ}$ ) to blob $A\left(\mathrm{PA}-65^{\circ}\right)$, obtaining a wide total opening angle of $90^{\circ}$.

The unidirectional asymmetry of IRS 1 in the NIR and MIR images (see Fig. 6) is naturally explained in this context as scattered light from the inner $(<1500 \mathrm{AU})$ walls of the cavity. This scenario is also consistent with the southeast-northwest orientation of the $\mathrm{CO}$ outflow, aligned roughly parallel to the methanol masers $\left(\mathrm{PA} \sim-62^{\circ}\right.$ ). Blobs $A, B, B^{\prime}$ are located within the same direction and might resemble either clumps in the cavity or recent ejecta from the outflow. The various blobs might also indicate the presence of several outflows.

In order to resolve the misalignment of the radio-continuum core with respect to the other outflow tracers, it was proposed that the radio-continuum emission might arise from a photoevaporated disk wind (Lugo et al. 2004).

However, as noted above, the methanol maser feature A lacks a quantitative modeling up to now.

\subsection{Scenario B: precessing jet model}

\subsubsection{Constraints from the methanol maser disk}

The circumstellar-disk modeling presented by Pestalozzi et al. (2004) reproduces the observational data for maser feature A in minute detail. Assuming a central mass of $30 M_{\odot}$ and Keplerian rotation, this model confines the inner $\left(r_{i} \sim 290 \mathrm{AU}\right)$ and outer $\left(r_{\mathrm{O}} \sim 750 \mathrm{AU}\right)$ radii of the disk (these radii scale as $\left(M / 30 M_{\odot}\right)^{-1 / 3}$ with the central mass $\left.M\right)$. The model does not set strict constraints on the inclination and orientation of the disk on the sky.

An uncertainty in the disk inclination arises from the assumption that methanol is formed within a surface layer of the disk from photoevaporation of $\mathrm{H}_{2} \mathrm{O}$. The midplane of the disk might therefore be inclined within certain limits. A distinct inclination is suggested by the fact that the NIR/MIR continuum emission, as well as the $\mathrm{H}_{2}$ shock tracer line emission, appears more pronounced towards the north than towards to south (see Figs. 1 and $6 \mathrm{~h}$ ). An inclination of the northern outflow towards us is also indicated by $\mathrm{CO}$ outflow observations (e.g. Kameya et al. 1989), which show the blueshifted CO lobe of IRS1 towards the northwest (see Figs. 6f and g).

The disk orientation on the sky can only be constrained by the maser observations with a limited accuracy since the masers only trace a narrow latitudinal arc of the disk, missing potential disk warping. Nevertheless, it is still reasonable to identify the disk orientation with the linearly-aligned feature A.

\subsubsection{Indications for disk and jet precession}

Assuming that the alignment of the masers is representative of the orientation of the disk midplane (i.e. assuming disk warping is negligible ${ }^{4}$ ), it is evident that the direction perpendicular to the disk plane (PA $+19^{\circ}$, the expected outflow direction) is significantly misaligned from the axes of the bipolar $\mathrm{CO}$ outflow and the NIR fan-shaped region (PA $\sim-20^{\circ}$; illustrated within Fig. 3). Also, the observed bending (Campbell 1984; Gaume et al. 1995) in the radio continuum could indicate a change in the outflow direction. Whereas the inner core $\left.(\$ 0)^{\prime} 5\right)$ is orientated along PA $\sim 0^{\circ}$, the outer core $\left(00^{\prime \prime} 5-1^{\prime \prime} .0\right)$ bends slightly towards the west $\left(\mathrm{PA} \sim-25^{\circ}\right)$. This might indicate that the outflow changed its direction by this amount within the times needed by the jet to propagate the appropriate projected distances $(\sim 25$ and $\sim 50$ years).

The bending detectable in the UC H II region on scales of $\$ 1$ '. 0 seems to continue at larger scales within the morphology observed in our speckle images, suggesting an S-shaped fine-structure of the diffuse emission extending from IRS1 initially towards the northwest and further out towards north. The blobs $A, B$, and $B^{\prime}$ observed close to IRS1 (PA $\sim-60^{\circ}$ ) might represent the most recent ejecta, whereas the weak features which appear further away in our images $(C, D)$ might trace earlier epochs of the history of the outflow.

Based on these indications, we suggest a disk and jet precession model. The fan-shaped diffuse emission in which the S-structure is embedded can be explained as scattered light from the walls of an outflow cavity, which was cleared by the proposed wandering jet.

The western wall of this wide, carved-out outflow cavity might appear within our NIR and the MIR images as an elongation of IRS1. Since this elongation extends mainly towards the northwest, there must be an additional reason why the western wall of this cavity appears more prominent than the eastern wall. A possible explanation might be shock excitation of the western wall, which would cool through emission in shock tracer lines like $\mathrm{H}_{2}$, which is contributing to the recorded $K^{\prime}$-band.

\footnotetext{
4 Interestingly, the converse assumption (that disk warping is nonnegligible) implies disk precession as well, as the jet would be launched perpendicular to the warped surface of the inner part of the disk.
} 

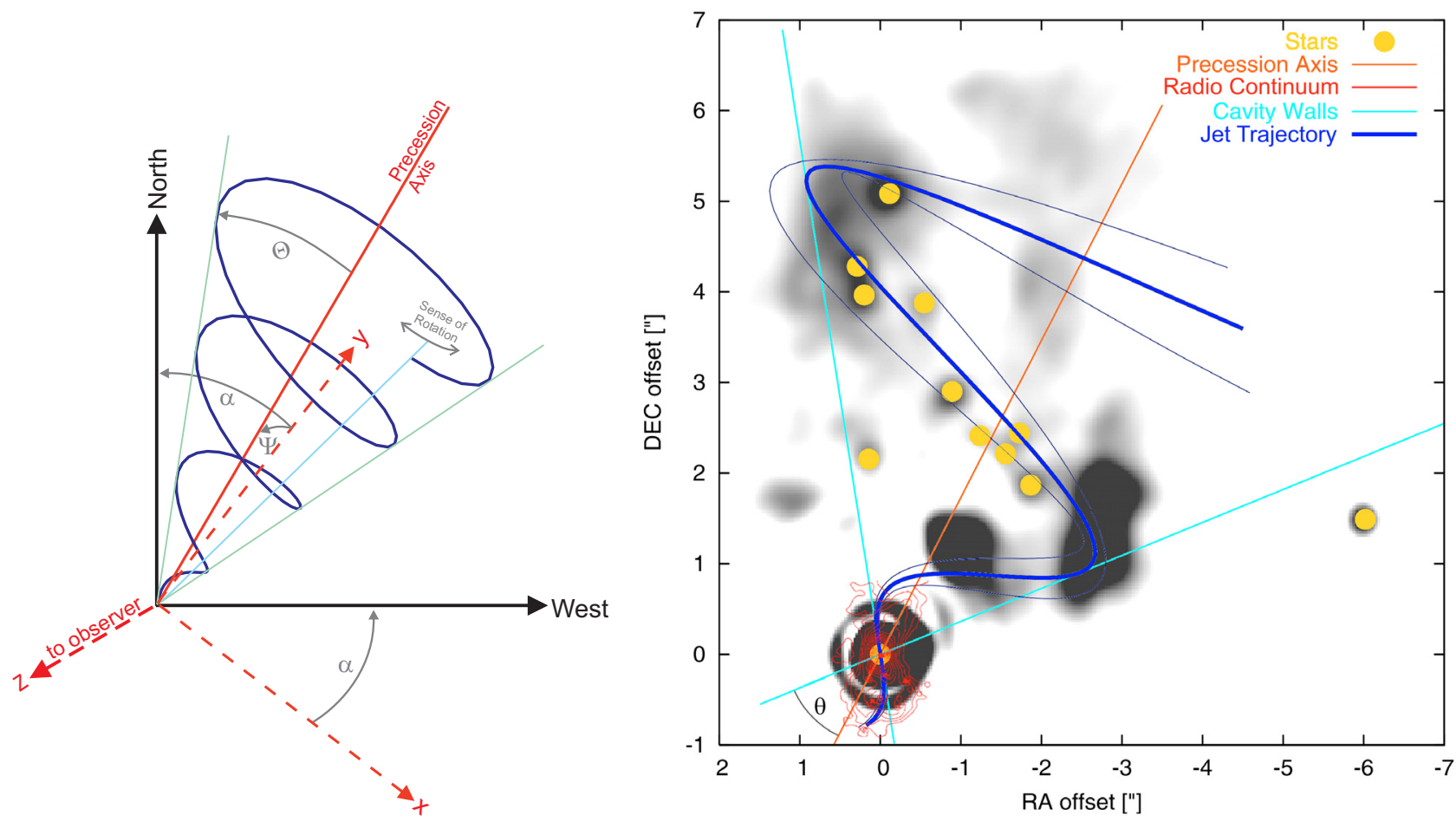

Fig. 7. Left: illustration of the analytic precession model presented in Sect. 4.4.3. Right: MMT speckle image overplotted with the trajectory of ejecta from a precessing outflow projected onto the plane of the sky (thick blue line) as described by the analytic precession model. For the counter-clockwise precession, the parameters $P_{\text {prec }}=280 \mathrm{yr}, \Theta=40^{\circ}, \alpha=-30^{\circ}, \psi=0^{\circ}$ (precession axis within the plane of the sky), and $\phi_{0}=0^{\circ}$ were used. In order to simulate a finite collimation of the flow, we varied both $\alpha$ and $\psi$ by $\pm 5^{\circ}$, yielding the trajectory given by the thin blue lines. The red contours show the $15 \mathrm{GHz}$ radio continuum map by Hutawarakorn \& Cohen (2003; using data by Gaume et al. 1995).

Assuming the precession period derived in Sect. 4.4.3, the outflow (which currently points towards PA $\sim 19^{\circ}$ ) would have excited the western wall of this cavity $\sim 140$ years ago, which corresponds roughly to the $\mathrm{H}_{2}$ radiative cooling time.

The arrangement of the fainter cluster members embedded within the diffuse emission can be understood in this context, too: Taking into account that IRS1 is still deeply embedded in its natal circumstellar cloud, the jet would have cleared the envelope along its wandering path. The decreasing column density results in lower extinction along the jet's path, revealing the fainter stars which likely formed in the vicinity of IRS1. The fainter stars might therefore be detectable only in those regions where the precessing jet reduces the extinction sufficiently. Within the blobs closest to IRS1, stars may be undetectable because of either inclination effects or confusion with the significantly higher surface brightness of blobs IRS $1 A, B$, and $B^{\prime}$ (limiting the sensitivity to detect point sources), or because of the high density of the outflowing material itself, providing intrinsic extinction.

The outflow tracers observed at rather large scales $\left(\mathrm{CO}, \mathrm{H}_{2}\right.$, see Fig. 6) are oriented roughly in the same direction as the NIR fan-shaped structure. The CO channel maps by Scoville et al. (1986; Fig. 6g) suggest a change in the orientation of the $\mathrm{CO}$ outflow lobes for low and high velocities. Whereas the low velocity $\mathrm{CO}$ outflow is oriented along the east-west direction, the high velocity lobes are oriented along $\mathrm{PA} \sim-35^{\circ}$. As $\mathrm{CO}$ traces material swept-up by the outflow and has a relatively long cooling time (of the order of $10^{4} \mathrm{yr}$ ), the different orientations observed at low- and high velocities are more difficult to interpret.

Finally, we speculate that the precession model might also explain why the velocities of the methanol maser features B, C, $\mathrm{D}$, and $\mathrm{E}$ are in the same range as the velocities of the $\mathrm{CO}$ outflow (De Buizer \& Minier 2005), but show opposite signs for the LOS velocity with respect to feature A (maser features B, $\mathrm{C}, \mathrm{D}$, and $\mathrm{E}$ are blue-shifted, whereas the southern $\mathrm{CO}$ lobe is red-shifted). Assuming precession, the $\mathrm{CO}$ outflow would trace the average outflow direction around the precession axis (with the southern axis oriented away from the observer), whereas the methanol masers might trace clumps very close to the source, which were excited more recently when the southern part of the outflow was pointing towards the observer ${ }^{5}$.

In general, precession can explain the change in the flow orientation, but potential alternative explanations include density gradients in the surroundings of IRS1, the presence of multiple outflows, and flow deflection.

\subsubsection{Analytic precession model}

In order to get a rough estimate for the precession parameters, we employ a simple analytical model with constant radial outflow speed $v$. On the radial motion we superpose a precession with period $P_{\text {prec }}$, leading to the wave number

$k=\frac{2 \pi}{v P_{\mathrm{prec}}}$

by the time that ejected material travels a distance $r$ from the source, the direction of the jet axis changes by the angle $k r$.

To describe the jet propagation in three dimensions we introduce a Cartesian coordinate system centered on IRS1 whose $z$-axis is along the line of sight (see Fig. 7, top). The precession

5 This is consistent with the precession parameters determined in Sect. 4.4.3, where we find that the half-opening angle of the precession $\Theta$ is larger than the inclination of the precession axis with respect to the plane of the sky $\psi$. 
axis is in the $y-z$ plane inclined by angle $\psi$ to the plane of the $\mathrm{sky}^{6}$, and the jet axis makes an angle $\Theta$ with it. For counterclockwise $^{7}$ precession, the coordinates of material at distance $r$ from the origin are

$$
\left(\begin{array}{l}
x \\
y \\
z
\end{array}\right)=r \times\left(\begin{array}{l}
\sin \Theta \cos \phi \\
\cos \Theta \cos \psi+\sin \Theta \sin \phi \sin \psi \\
-\cos \Theta \sin \psi+\sin \Theta \sin \phi \cos \psi
\end{array}\right)
$$

where $\phi=\phi_{0}+k r$ is the jet's azimuthal angle from the $x$-axis. The initial phase $\phi_{0}$ can be taken as $0^{\circ}$ since the direction perpendicular to the methanol feature A seems to coincide with the eastern wall of the outflow cavity. The PA $\alpha$ of the $y$-axis can be set from the average angle of the fan-shaped region in the speckle images as $\alpha=-30^{\circ}$. Using $v=250 \mathrm{~km} \mathrm{~s}^{-1}$, as measured by Gaume et al. (1995) within the H66 $\alpha$ recombination line, leaves as free parameters $P_{\text {prec }}, \Theta, \psi$, and the sense of rotation. Trying to fit the orientation of the maser disk, the orientation of the UC H II region, and the position of the NIR blobs with these parameters simultaneously, we find reasonable agreement with a precession period $P_{\text {prec }}=280 \pm 10 \mathrm{yr}$, a precession angle $\Theta=40^{\circ} \pm 3^{\circ}$, a counter-clockwise sense of rotation, and small inclination $\psi=0^{\circ} \pm 10^{\circ}$. At larger inclination angles $\left(\gtrsim 10^{\circ}\right)$ loops start to appear, significantly degrading the agreement. In Fig. 7, we show the projected trajectory of the proposed wandering jet with the thick line, whereas the thin lines give the path obtained with a variation of $\pm 5^{\circ}$ in $\alpha$ and $\psi$, resembling the finite width of the flow.

The analytic model presented in this section might suffice in order to get a rough estimate of the precession parameters, although it does not take into account the interaction of the flow with the ambient medium nor the excitation and cooling of the ambient material.

These parameters can be used to predict how the orientation of the methanol maser disk changes with time. Using the PA at the phase $\phi_{0}=0^{\circ}$ as reference, one expects that the PA changes only marginally (less than $1^{\circ}$ ) within $10 \mathrm{yr}$. A much more significant change of $10^{\circ}\left(20^{\circ}\right)$ would be expected after $36 \mathrm{yr}(50 \mathrm{yr})$, which would be detectable with future VLBI observations.

\subsubsection{Numerical molecular hydrodynamic simulations}

A large number of studies about the structure and evolution of precessing protostellar jets can be found in literature (e.g. Raga \& Biro 1993; Völker et al. 1999; Raga et al. 2004; Rosen \& Smith 2004; Smith \& Rosen 2005a), although most of these studies focus on jets from low-mass stars with rather narrow precession angles. As the number of simulations carried out for wide precession angles is much more limited (e.g. Cliffe et al. 1996), we performed a new hydrodynamic simulation. Besides the general morphology, we aim for comparing the position of the newly discovered fainter stars with the column density variations caused by a precessing jet, which was beyond the scope of earlier studies.

We use the version of the ZEUS-3D code as modified by Smith \& Rosen (2003), which includes some molecular cooling and chemistry, as well as the ability to follow the molecular

\footnotetext{
${ }^{6}$ Positive values of $\psi$ indicate an inclination out of the plane of the sky towards the observer.

${ }^{7}$ For the sense of rotation, we follow the convention that counterclockwise rotation (as measured from the source along the precession axis) corresponds to a positive sign of the phase $\phi$.
}

$\left(\mathrm{H}_{2}\right)$ fraction. The large precession envisioned for the flow associated with NGC 7538 IRS1 requires that the simulation be performed on a very wide computational grid. Due to computational limits, we were restrained to use for this simulation a 3D Cartesian grid of 275 zones in each direction, where each zone spans $2 \times 10^{14} \mathrm{~cm}$ in each direction. This grid balances the desire for some spatial resolution of the flow with the ability to simulate a sufficiently large part of the observed flow associated with NGC 7538 IRS1. Still, the total grid size $(\sim 0.018 \mathrm{pc})$ is smaller than the projected distance between IRS 1 and $K^{\prime}$-band feature $C$.

Owing to the rather small physical size of the grid, we have chosen a nominal speed of $150 \mathrm{~km} \mathrm{~s}^{-1}$, reduced from the inferred value of $250 \mathrm{~km} \mathrm{~s}^{-1}$ for this source. The flow is precessed with a nearly $30^{\circ}$ precession angle, with the amplitude of the radial components of the velocity 0.55 of that of the axial component. The precession has a period of 120 years, which leads to $1.25 \mathrm{cy}-$ cles during a grid crossing time. The flow is also pulsed, with a $30 \%$ amplitude and a 30 year period. This short period assists in the reproduction of the multiple knots of $K^{\prime}$-band emission near NGC 7538 IRS1. The jet flow also is sheared at the inlet, with the velocity at the jet radius 0.7 that of the jet center. We have chosen a jet number density of $10^{5}$ hydrogenic nuclei $\mathrm{cm}^{-3}$, while the ambient density is $10^{4}$. The simulated jet radius is $4.0 \times 10^{15} \mathrm{~cm}(20$ zones $)$. Thus, the time-averaged mass flux is $2.6 \times 10^{-6} M_{\odot} \mathrm{yr}^{-1}$, which is three orders of magnitude lower than the value as determined for the $\mathrm{CO}$ outflow (Davis et al. 1998). Similar calculations of the momentum flux and kinetic energy flux, or mechanical luminosity, yield values of $3.8 \times$ $10^{-4} \mathrm{~km} \mathrm{~s}^{-1} M_{\odot} \mathrm{yr}^{-1}$ and $4.7 L_{\odot}$, respectively.

After the convolution with a PSF resembling the resolution obtained in real observations the $\mathrm{H}_{2}$ emission in our simulation shows a morphology which is similar to the one seen in the $K^{\prime}$ band speckle image. In particular, the simulations might also explain features $D$ and $E$ as associated with the proposed precessing jet (compare Figs. 8a with 3 ). The simulations also show that the $\mathrm{CO}$ emission, which can be expected for a precessing jet at larger distances from the driving source, appears very smooth, which is also in accordance with the $\mathrm{CO}$ observations made for NGC 7538 IRS1.

We note that the fainter stars $e$ to $n$ reported in Sect. 3.1.3 are located in the region where the column density in our precessing jet simulation appears particularly low (see Fig. 8c, left column), supporting the scenario proposed in Sect. 4.4.2.

\subsubsection{Possible precession mechanisms}

Several mechanisms have been proposed which can cause jet bending or jet precession, although most of them were established for low- and intermediate stars and can cause precession angles of only a few degrees (Fendt \& Zinnecker 1998; Eislöffel $\&$ Mundt 1997). For the case of high-mass stars and larger precession angles $\left(\Theta \sim 40^{\circ}\right)$, Shepherd et al. (2000) summarized the three most promising concepts that could induce precession into circumstellar disks. We discuss how well these mechanisms can explain the observations of IRS1. For all cases, it is assumed that the outflow is launched close to the center of the disk and that a precession of the inner parts of the disk will translate into a precession of the collimated flow (Bate et al. 2000).

1. Radiative-induced warping: Armitage \& Pringle (1997) suggested that geometrically thin, optically thick accretion disks can become unstable to warping if the incident radiation from the stellar source is strong enough. As this warping 

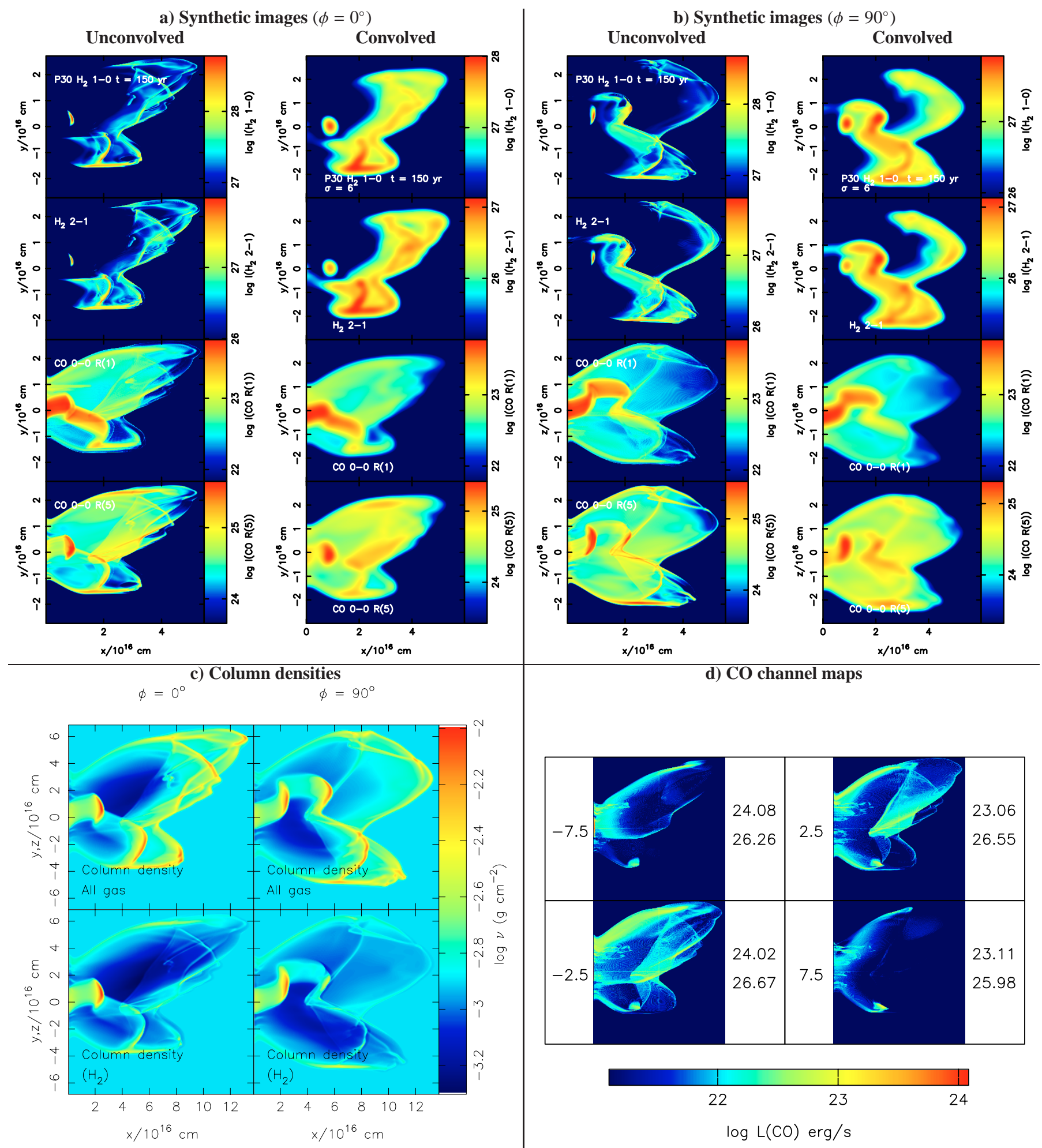

Fig. 8. a), b): Synthetic images $\left(\mathrm{H}_{2} 1 \rightarrow 0,2 \rightarrow 1\right.$; $\left.\mathrm{CO} \mathrm{R}(1), \mathrm{R}(5)\right)$ from our ZEUS-3D molecular hydrodynamic simulation, shown for two different projections. In a) the images are also shown after a convolution with a Gaussian which roughly resembles the resolution obtained in our speckle observation (Fig. 3). c) Shows the total gas column density and the $\mathrm{H}_{2}$ column density for the same projections. Finally, in d) we show channel maps of the $\mathrm{CO}$ outflow for four velocity bins. Each velocity bin has a width of $5 \mathrm{~km} \mathrm{~s}^{-1}$ and the central velocity is given by the number on the left of each image (in $\mathrm{km} \mathrm{s}^{-1}$ ). The two numbers on the right of each image indicate the log of the maximum integrated luminosity in any single element in the image (Top, in $\mathrm{erg} \mathrm{s}^{-1}$ ) and the log of the total integrated luminosity in the entire velocity bin (Bottom). The angle $\phi$ gives the angle between the $z$ axis and the LOS, corresponding to a rotation around the precession axis ( $x$ axis). Please see the text (Sect. 4.4.4) for a description of the complete model parameters.

instability is expected to occur only at disk radii larger than a critical radius $R_{\text {crit }}$, we can estimate whether radiative-induced disk warping is expected at the inner part of the IRS1 disk. Using a stellar mass of $M \sim 30 M_{\odot}$ (Pestalozzi et al. 2004), 
a mass accretion rate of the order of the mass outflow rate $\dot{M}_{\text {acc }} \approx \dot{M}_{\text {outflow }} \approx 5.4 \times 10^{-3} M_{\odot} \mathrm{yr}^{-1}$ (Davis et al. 1998), and a luminosity $L \approx 9.6 \times 10^{4} L_{\odot}$ (Akabane \& Kuno 2005), we use Eq. (5) by Armitage \& Pringle (1997) and the assumptions listed in Shepherd et al. (2000) and obtain a critical radius $R_{\text {crit }} \sim 200$ pc. Since this is far beyond the inner edge of the disk where the jet collimation is expected to happen, it is very unlikely that the radiation emitted by the star or due to accretion causes any noticeable warping within the disk.

2. Anisotropic accretion events: the impact/merging of (low mass) condensations can change the orientation of the disk angular momentum vector. In such a dramatic event, angular momentum can be transferred from the impactor onto the accretion disk, potentially resulting in a net torque in the rotation of the disk. To estimate the precession angle, which could result from anisotropic accretion, very detailed assumptions about the disk, the impacting condensation, and their kinematics must be made. Since no data is available to estimate these quantities, we refer to the example computed by Shepherd et al. (2000) and note that in extreme cases, such an accretion event could cause a sufficiently large precession angle in the case of NGC 7538 IRS1 as well. However, in this scenario one would expect rather sudden changes in the jet direction rather than a smooth precession.

3. Tidal interactions with a companion: warping and precession of the disk could be caused by tidal interactions with one or more companions on non-coplanar orbits. We assume the simplest case of a binary: with stellar masses $M_{\mathrm{p}}$ (primary) and $M_{\mathrm{s}}$ (secondary), an orbit with inclination $i$ with respect to the disk plane, and a semimajor axis $a$. The mass ratio shall be denoted $q=M_{\mathrm{p}} / M_{\mathrm{s}}$ and will be assumed as unity. Our observations place an upper limit on the separation of such a companion (see Fig. 4). Two cases can be considered:

3a) circumprimary disk $\left(\boldsymbol{a}>\boldsymbol{r}_{\mathbf{0}}\right)$ : because tidal torques would truncate the disk at about 0.3 times the binary separation (Lubow \& Ogilvie 2000), we obtain a lower limit for the binary separation (for a circular orbit), namely, $a>2500$ AU. However, a binary with such a large separation would be not suited to explain the observations since the orbital period would be $>2 \times 10^{4} \mathrm{yr}\left(M_{\mathrm{p}}+M_{\mathrm{s}}=30 M_{\odot}\right)$, implying a disk precession rate of $>4 \times 10^{5} \mathrm{yr}$ (Bate et al. 2000). Assuming an extreme eccentricity might yield a short precession period of the order of $10^{2} \mathrm{yr}$ but implies strong, periodic interactions between the companion and the disk during each perihelion passage. As this would quickly distort and truncate the disk, we see this assumption contradicts the methanol maser structure, which suggests a smooth extension of the methanol layer from $\sim 290$ AU to $\sim 750$ AU.

3b) circumbinary disk $\left(\boldsymbol{a}<\boldsymbol{r}_{i}\right)$ : smoothed particle hydrodynamic simulations by Larwood \& Papaloizou (1997) showed that a binary on a non-coplanar orbit with large inclination $i$ could cause strong quasi-rigid body precession of the circumbinary disk (for $q>10$ ) and strong warping, especially on the inner edge of the disk $(q \sim 1)$. The same authors report that the disk precession frequency $\omega_{\text {prec }}$ should be lower than the orbital frequency of the binary $\omega_{\text {binary }}$. To make an order-of-magnitude estimation for the orbital period that would be expected for this hypothetical IRS 1 binary system, one can assume $\omega_{\text {prec }} \approx$ $\omega_{\text {binary }} / 20$ (Bate et al. 2000 ) to obtain $P_{\text {binary }} \approx 14 \mathrm{yr}$ for the binary period, corresponding to a separation of $a_{\text {binary }} \approx 19 \mathrm{AU}$ ( $\sim 7$ mas). This binary separation then puts a lower limit on the radius of the inner edge of the circumstellar disk. As this scenario can trigger the fast disk precession without truncating the extended disk structure traced by the methanol masers, we consider a circumbinary disk as the most plausible explanation.

\subsection{The IRS2 companion and flow interaction with the IRS2 UC HII region}

The spectral type of IRS2 was estimated to be O4.5 (Akabane \& Kuno 2005), corresponding to a luminosity of $\sim 6.4 \times 10^{5} L_{\odot}$. Using the measured $K^{\prime}$-band flux ratio, one can make rough estimates for the spectral type of the two components reported in Sect. 3.1.2. By assuming the total luminosity is attributed only to the two components, we obtain a spectral type of O5 for IRS $2 a$ and O9 for IRS $2 b$ (using the OB star luminosities from Vacca et al. 1996).

Within our images, the wide-opening angle outflow cone from IRS1 seems to extend well out to IRS2. This offers an explanation for the shock tracer line emitting region that was imaged around IRS2 (Bloomer et al. 1998; see Fig. 6i). The bowshock-like morphology of the $[\mathrm{Fe} \mathrm{II}]$ and $\mathrm{H}_{2}$ emission suggests that the shock is excited from the south (which is roughly the direction towards IRS1). In the direction opposite IRS1, the $[\mathrm{Fe} \mathrm{II}]$ and $\mathrm{H}_{2}$ emission even shows a cavity-like structure, which also appears in the $6 \mathrm{~cm}$-radio continuum. Bloomer et al. (1998) suggested a stellar wind bowshock scenario, in which IRS2 moves with a speed of $\sim 10 \mathrm{~km} \mathrm{~s}^{-1}$ towards the southwest through the ambient molecular cloud. We note that the morphology could also be explained by interaction between the IRS1 outflow and IRS2 outflows. Based on its young age, IRS2 might also launch a powerful wind itself, causing the distinct shock zone which appears within the shock tracer emission (see Fig. 6i) and which is also detectable in our $K^{\prime}$-band image (arc-like morphology between features $G$ and $H$ ).

\subsection{Outflow structures from IRS1 at larger spatial scales}

Figures 6a to $d$ shows mosaics of the vicinity of IRS1 in the four IRAC bands. Although IRS1 and IRS2 appear saturated in these images (shown in logarithmic scaling) and banding (vertical and horizontal stripes produced by IRS1 and IRS9) appears especially in the 5.8 and $8.0 \mu \mathrm{m}$ images, structures potentially related to IRS1 can be observed. About 40" towards the southeast of IRS1, a bowshock structure can be seen, which is also present in the $\mathrm{H}_{2}$ image by Davis et al. (1998). This bowshock points in the same direction as the redshifted lobe of the $\mathrm{CO}$ outflow (see Fig. 6f) and just opposite to the outflow direction identified in our speckle image at small scales. Thus, it is possible that this bow traces the southeastern part of the IRS1 outflow. In our speckle image, the inner part of this southeastern outflow is not visible; likely a result of strong intervening extinction.

Furthermore, it is interesting to note that the "ridge" connecting the IRS1-3 cluster with IRS4 and IRS5 follows the western wall of the outflow direction identified in our speckle image (see top of Fig. 6). It is possible that the total extent of the IRS1 outflow also reaches much further northwest than the structure seen in the speckle image, contributing to the excitation of the western part of the bubble seen in the IRAC bands and the shocks in the $\mathrm{H}_{2}$ image by Davis et al. (1998; see Fig. 6h). 


\section{Evidence for triggered star formation in the NGC 7538 star forming region}

It has been proposed by many authors that star formation seems to propagate southeastwards throughout the NGC 7538 complex (Werner et al. 1979; McCaughrean et al. 1991; Ojha et al. 2004). This is indicated by the spatial arrangement of the members of this star forming region, which also seems to agree with the expected evolutionary sequence: starting about 3 ' northwest of IRS1, O stars located in the H II region represent the most developed evolutionary state, followed by the IRS1-3 cluster and their associated UCH II regions, with the compact reflection nebula around IRS9 representing the youngest member of this star formation site. In agreement with this picture, Balog et al. (2004) measured the reddening of stars throughout NGC 7538 and found a gradient in reddening with the most heavily reddened sources in the southeast.

The presented IRAC images can also be interpreted in support of this scenario since the "ridge"-like feature connecting IRS1-3, IRS4, IRS5 seems to trace the interface between the northeastern bubble (visible at NIR/MIR wavelengths) and the submillimeter bubble, which appears in the $450 \mu \mathrm{m}$ and $850 \mu$ m-maps by Reid \& Wilson (2005; see Fig. 2). This suggests that in NGC 7538, star formation was triggered by the compression of gas just at the interface layer of these expanding bubbles, sequentially initiating the formation of the observed chain of infrared sources.

Ojha et al. (2004) suggested that IRS6, the most luminous source in the NGC 7538 region, might be the main exciting source responsible for the optical H II region. Inspecting the IRAC color composites, this scenario is supported by the morphology of the bright, curved structure west of IRS6. In the IRAC $8 \mu \mathrm{m}$ band (red in Fig. 2c), this structure appears particularly prominent. As it is known that emission in this IRAC band is often associated with PAHs, this suggests that this region is illuminated by strong UV radiation from IRS6. Other features, such as the conical structure around 2MASS $23135808+6130484$ and the structure northeast of IRS7, also show a symmetry towards IRS6.

\section{Summary and conclusions}

Bispectrum speckle interferometry and archival Spitzer/IRAC imaging of the massive protostars NGC 7538 IRS1/2 and their vicinity are presented. We summarize our results as follows:

1. The clumpy, fan-shaped structure seen in our speckle images most likely traces recent outflow activity from IRS1, consistent with the direction of the blue-shifted lobe of the known $\mathrm{CO}$ outflow. A bowshock structure noticeable in the IRAC images $\sim 40^{\prime \prime}$ southeast of IRS1 suggests that the total extent of the outflow might be several parsecs. The outflow might have also contributed to shaping and exciting the bubble-like structure, which is prominent in all four IRAC bands (although contributions from several other sources, especially IRS6, are also evident).

2. A companion around the high-mass star NGC 7538 IRS2 was discovered. Furthermore, we see indications for interactions between the IRS1 flow and outflows or stellar winds from IRS2 (nebulosity surrounding IRS2).

3. A jet precession model seems suitable to describe the features observed within our NIR images, simultaneously explaining the misalignment between the putative methanol maser disk, the UCH II region, and the outflow tracers detected at larger scales (CO). A simple analytic precession model was used to extract order-of-magnitude estimates for the precession parameters. Using these we estimate tidal interaction of a close binary system with a circumbinary disk as the most plausible gyroscopic mechanism, which is triggering the precession.

4. The presented molecular hydrodynamic simulations can reproduce some of the fine-structure observed in our NIR images and indicate that the arrangement of the detected fainter stars might be explained as a column-density effect, caused by the proposed precessing jet.

5. The prominent sites of ongoing high-mass star formation in NGC 7538 seem to be located just at the interface between two bubble-like structures - one is visible in the presented IRAC images, the other traced by submillimeter observations. The gas compression caused by the expansion of these bubbles might have triggered star formation in this region.

While it is well established that the outflows of HMPOs generally appear less collimated than those of their low-mass counterparts, the recent discovery of evidence for outflow precession for an increasing number of massive YSOs might indicate a common launching mechanism for all outflow driving sources of all stellar masses. The observed widening in HMPO outflows might be due to selection effects (Shepherd 2005) and/or precession of a collimated jet. The large precession angles reported for IRAS 20126+4104 (Shepherd et al. 2000), S140 IRS1 (Weigelt et al. 2002), IRAS 23151+5912 (Weigelt et al. 2005), and now NGC 7538 IRS1 (this paper) might point towards a rather dramatic precession mechanism, maybe the presence of very close, high-mass companions on non-coplanar orbits.

We strongly encourage further observations of IRS1, especially to detect potential companions either by near-infrared long-baseline interferometry or radial velocity measurements.

Acknowledgements. We thank all the participants of the NGC 7538 collaboration for very fruitful discussions that contributed to the achievement of this paper. The collaboration consists of Roy Booth, John Conway, James De Buizer, Moshe Elitzur, Stefan Kraus, Vincent Minier, Michele Pestalozzi, and Gerd Weigelt. We also acknowledge the BTA and MMT staff for their support of this run, and D. Apai and I. Pascucci for assistance during the MMT observations.

S.K. was supported for this research through a fellowship from the International Max Planck Research School (IMPRS) for Radio and Infrared Astronomy at the University of Bonn.

The numerical hydrodynamic simulations were executed on the Armagh SGI Origin 2000 computer (FORGE), acquired through the Particle Physics and Astronomy Research Council (PPARC) JREI initiative with SGI participation. This work is based in part on archival data obtained with the Spitzer Space Telescope, which is operated by the Jet Propulsion Laboratory, California Institute of Technology under a contract with NASA.

This publication makes use of data products from the Two Micron All Sky Survey, which is a joint project of the University of Massachusetts and the Infrared Processing and Analysis Center/California Institute of Technology, funded by the National Aeronautics and Space Administration and the National Science Foundation.

\section{References}

Akabane, K., \& Kuno, N. 2005, A\&A, 431, 183

Alvarez, C., Hoare, M., Glindemann, A., \& Richichi, A. 2004, A\&A, 427, 505 Armitage, P. J., \& Pringle, J. E. 1997, ApJ, 488, L47

Balog, Z., Kenyon, S. J., Lada, E. A., et al. 2004, AJ, 128, 2942

Bate, M. R., Bonnell, I. A., Clarke, C. J., et al. 2000, MNRAS, 317, 773

Blitz, L., Fich, M., \& Stark, A. A. 1982, ApJS, 49, 183

Bloomer, J. D., Watson, D. M., Pipher, J. L., et al. 1998, ApJ, 506, 727

Campbell, B. 1984, ApJ, 282, L27

Campbell, B., \& Persson, S. E. 1988, AJ, 95, 1185

Churchwell, E., Whitney, B. A., Babler, B. L., et al. 2004, ApJS, 154, 322

Cliffe, J. A., Frank, A., \& Jones, T. W. 1996, MNRAS, 282, 1114

Davis, C. J., Moriarty-Schieven, G., Eislöffel, J., Hoare, M. G., \& Ray, T. P. 1998, AJ, 115, 1118 
De Buizer, J. M., \& Minier, V. 2005, ApJ, 628, L151

Dickel, H. R., Rots, A. H., Goss, W. M., \& Forster, J. R. 1982, MNRAS, 198, 265

Dyck, H. M., \& Capps, R. W. 1978, ApJ, 220, L49

Dyck, H. M., \& Lonsdale, C. J. 1979, AJ, 84, 1339

Eislöffel, J., \& Mundt, R. 1997, AJ, 114, 280

Elsaesser, H., Birkle, K., Eiroa, C., \& Lenzen, R. 1982, A\&A, 108, 274

Fazio, G. G., Hora, J. L., Allen, L. E., et al. 2004, ApJS, 154, 10

Fendt, C., \& Zinnecker, H. 1998, A\&A, 334, 750

Fischer, J., Sanders, D. B., Simon, M., \& Solomon, P. M. 1985, ApJ, 293, 508

Franco-Hernández, R., \& Rodríguez, L. F. 2004, ApJ, 604, L105

Gaume, R. A., Goss, W. M., Dickel, H. R., Wilson, T. L., \& Johnston, K. J. 1995, ApJ, 438, 776

Hackwell, J. A., Grasdalen, G. L., \& Gehrz, R. D. 1982, ApJ, 252, 250

Hofmann, K.-H., \& Weigelt, G. 1986, A\&A, 167, L15

Hoffman, I. M., Goss, W. M., Palmer, P., \& Richards, A. M. S. 2003, ApJ, 598, 1061

Hora, J. L., Fazio, G. G., Allen, L. E., et al. 2004, in Microwave and Terahertz Photonics. Edited by Stohr, Andreas; Jager, Dieter; Iezekiel, Stavros, ed. J. C. Mather, Proc. SPIE, 5487, 77

Hutawarakorn, B., \& Cohen, R. J. 2003, MNRAS, 345, 175

Johnston, K. J., Stolovy, S. R., Wilson, T. L., Henkel, C., \& Mauersberger, R. 1989, ApJ, 343, L41

Kameya, O., Hasegawa, T. I., Hirano, N., Takakubo, K., \& Seki, M. 1989, ApJ, 339,222

Kameya, O., Morita, K.-I., Kawabe, R., \& Ishiguro, M. 1990, ApJ, 355, 562

Kawabe, R., Suzuki, M., Hirano, N., et al. 1992, PASJ, 44, 435

Krumholz, M. R., McKee, C. F., \& Klein, R. I. 2005, ApJ, 618, L33

Labeyrie, A. 1970, A\&A, 6, 85

Larwood, J. D., \& Papaloizou, J. C. B. 1997, MNRAS, 285, 288

Lohmann, A. W., Weigelt, G., \& Wirnitzer, B. 1983, Appl. Opt., 22, 4028

Lubow, S. H., \& Ogilvie, G. I. 2000, ApJ, 538, 326

Lugo, J., Lizano, S., \& Garay, G. 2004, ApJ, 614, 807

Lynds, B. T., \& O’Neil, E. J. 1986, ApJ, 306, 532

Madden, S. C., Irvine, W. M., Matthews, H. E., Brown, R. D., \& Godfrey, P. D. 1986, ApJ, 300, L79
McCaughrean, M., Rayner, J., \& Zinnecker, H. 1991, Mem. Soc. Astron. It., 62, 715

Menten, K. M., Walmsley, C. M., Henkel, C., et al. 1986, A\&A, 169, 271

Minier, V., Booth, R. S., \& Conway, J. E. 1998, A\&A, 336, L5

Minier, V., Booth, R. S., \& Conway, J. E. 2000, A\&A, 362, 1093

Ojha, D. K., Tamura, M., Nakajima, Y., et al. 2004, ApJ, 616, 1042

Pestalozzi, M. R., Elitzur, M., Conway, J. E., \& Booth, R. S. 2004, ApJ, 603, L113

Preibisch, T., Balega, Y., Hofmann, K.-H., Weigelt, G., \& Zinnecker, H. 1999, New Astronomy, 4, 531

Raga, A. C., \& Biro, S. 1993, MNRAS, 264, 758

Raga, A. C., Riera, A., Masciadri, E., et al. 2004, AJ, 127, 1081

Reid, M. A., \& Wilson, C. D. 2005, ApJ, 625, 891

Rosen, A., \& Smith, M. D. 2004, MNRAS, 347, 1097

Rots, A. H., Dickel, H. R., Forster, J. R., \& Goss, W. M. 1981, ApJ, 245, L15

Sandell, G., \& Sievers, A. 2004, ApJ, 600, 269

Scoville, N. Z., Sargent, A. I., Sanders, D. B., et al. 1986, ApJ, 303, 416

Shepherd, D. 2005, in IAU Symp., 237

Shepherd, D. S., Yu, K. C., Bally, J., \& Testi, L. 2000, ApJ, 535, 833

Smith, M. D., \& Rosen, A. 2003, MNRAS, 339, 133

Smith, M. D., \& Rosen, A. 2005a, MNRAS, 357, 579

Smith, M. D., \& Rosen, A. 2005b, MNRAS, 357, 1370

Tamura, M., Gatley, I., Joyce, R. R., et al. 1991, ApJ, 378, 611

Terquem, C., Eislöffel, J., Papaloizou, J. C. B., \& Nelson, R. P. 1999, ApJ, 512, L131

Vacca, W. D., Garmany, C. D., \& Shull, J. M. 1996, ApJ, 460, 914

Völker, R., Smith, M. D., Suttner, G., \& Yorke, H. W. 1999, A\&A, 343, 953

Weigelt, G., Balega, Y. Y., Preibisch, T., Schertl, D., \& Smith, M. D. 2002, A\&A, 381,905

Weigelt, G. P. 1977, Opt. Commun., 21, 55

Weigelt, G., \& Wirnitzer, B. 1983, Optics Letters, 8, 389

Weigelt, G., Beuther, H., Hofmann, K. ., et al. 2005, ArXiv Astrophysics e-prints Werner, M. W., Becklin, E. E., Gatley, I., et al. 1979, MNRAS, 188, 463

Wu, Y., Wei, Y., Zhao, M., et al. 2004, A\&A, 426, 503

Wynn-Williams, C. G., Becklin, E. E., \& Neugebauer, G. 1974, ApJ, 187, 473 